\title{
Time-domain implementation of an impedance boundary condition with boundary layer correction ${ }^{\dagger}$
}

\author{
E.J. Brambley*a and G. Gabard ${ }^{b}$ \\ Received 22 January 2016; Received in revised form 30 May 2016; \\ Accepted 31 May 2016; Available online 8 June 2016
}

\begin{abstract}
A time-domain boundary condition is derived that accounts for the acoustic impedance of a thin boundary layer over an impedance boundary, based on the asymptotic frequency-domain boundary condition of Brambley [2011, AIAA J. 49(6), pp. 1272-1282]. A finite-difference reference implementation of this condition is presented and carefully validated against both an analytic solution and a discrete dispersion analysis for a simple test case. The discrete dispersion analysis enables the distinction between real physical instabilities and artificial numerical instabilities. The cause of the latter is suggested to be a combination of the real physical instabilities present and the aliasing and artificial zero group velocity of finite-difference schemes. It is suggested that these are general properties of any numerical discretization of an unstable system. Existing numerical filters are found to be inadequate to remove these artificial instabilities as they have a too wide pass band. The properties of numerical filters required to address this issue are discussed and a number of selective filters are presented that may prove useful in general. These filters are capable of removing only the artificial numerical instabilities, allowing the reference implementation to correctly reproduce the stability properties of the analytic solution.
\end{abstract}

Keywords: Linearized Euler equations, acoustic impedance, inviscid boundary layer, absolute and convective instability.

\section{Introduction}

Since acoustic liners are routinely used within aeroengines to reduce noise, it is imperative that computational aeroacoustics (CAA) simulations include models of acoustic liners. In the frequency domain, acoustic liners are easily modelled as an impedance surface, where an oscillatory fluid pressure $\operatorname{Re}\left(p^{\prime} \exp \{i \omega t\}\right)$ at the surface gives rise to a normal fluid velocity $\operatorname{Re}\left(v_{s} \exp \{i \omega t\}\right)$ through the surface, linked through the complex impedance $Z(\omega)=p^{\prime} / v_{s}$. The entire physical modelling of the acoustic lining is encapsulated within the impedance $Z(\omega)$, for which numerous empirical and physical models exist [e.g. 2 [5] ; for further details, see Ref. 6 and references therein.

Typically in aeroacoustics, oscillations in the fluid are small perturbations to a steady mean flow, so that the total velocity is $\boldsymbol{u}_{0}(\boldsymbol{x})+\boldsymbol{u}^{\prime}(\boldsymbol{x}, t)$ and similarly for the pressure and density. The presence of mean flow complicates the application of an impedance boundary condition. For example, rather than setting $\boldsymbol{u}^{\prime} \cdot \boldsymbol{n}=p^{\prime} / Z$ at the boundary, where $\boldsymbol{n}$ is the normal to the acoustic lining, the boundary condition widely used is

$$
\mathrm{i} \omega \boldsymbol{u}^{\prime} \cdot \boldsymbol{n}=\left(\mathrm{i} \omega+\boldsymbol{u}_{0} \cdot \boldsymbol{\nabla}-\left(\boldsymbol{n} \cdot \boldsymbol{\nabla} \boldsymbol{u}_{0}\right) \cdot \boldsymbol{n}\right) p^{\prime} / Z .
$$

This equation is known as the Myers, or Ingard-Myers, boundary condition, after Ingard [7] and Myers [8], and corresponds to matching normal acoustic displacement between the fluid and the acoustic liner rather than normal velocity. For flat surfaces where $\left(\boldsymbol{n} \cdot \boldsymbol{\nabla} \boldsymbol{u}_{0}\right) \cdot \boldsymbol{n} \equiv 0$, equation (11) was shown by Eversman and Beckemeyer [9] and Tester [10] to be the correct limit of an infinitely thin inviscid boundary layer.

For CAA simulations the mean flow is assumed known (for example, from prior RANS calculations), and the small perturbations are to be calculated. Numerical schemes for calculating these small perturbations have different requirements from those used for calculating the steady flow in order to ensure

\footnotetext{
${ }^{\dagger}$ A preliminary version of some parts of this paper was presented as part of AIAA Paper 2013-2218 at the 19th AIAA/CEAS Aeroacoustics Conference in Berlin, Germany [1].

*Corresponding author. Tel.: +44 1223 760457. Fax.: +44 1223765900.

${ }^{a}$ Department of Applied Mathematics and Theoretical Physics, University of Cambridge, United Kingdom

${ }^{b}$ Institute of Sound and Vibration Research, University of Southampton, United Kingdom
} 
low dispersion and dissipation, and many optimized schemes exist [e.g. 11 13]. Requiring low dissipation means that instabilities are often found at under-resolved scales, of the order of half the Nyquist frequency (i.e. four points per wavelength spatially), necessitating selective filtering 13 15]. In time domain simulations, this selective filtering often takes the form of a weak low-pass spatial filter applied at every point at every time step.

For certain impedance models $Z(\omega)$, including the mass-spring-damper impedance [4, 16] for which $Z=R+\mathrm{i} m \omega-\mathrm{i} K / \omega$ and the Extended Helmholtz Resonator impedance [5, 17] for which $Z=R+\mathrm{i} m \omega-$ $\mathrm{i} \beta \cot (\omega L-\mathrm{i} \varepsilon / 2)$, time-domain versions of the frequency-domain boundary condition (11) are possible. However, high-frequency numerical instabilities are invariably present in time-domain simulations [4, 17, 18] when such impedances are used with slipping mean flow using the Myers boundary condition (10). These numerical instabilities are different from the instabilities due to the use of low-dissipation numerical schemes mentioned above [19]. Stabilizing the boundary condition requires the use of strong, wide-band filters to indiscriminately remove any form of instability. This is not surprising, since the underlying mathematical model being simulated is illposed [20] and supports arbitrarily quick exponential growth at arbitrarily short wavelengths. One way to regularize this problem is to consider a thin but non-zero thickness inviscid boundary layer over the lining. Since resolving this boundary layer, while possible, is computationally more expensive [e.g. 21] and introduces its own stability difficulties in the form of a continuous spectrum [22 24], it is convenient instead to modify the Myers boundary condition to include the effects of a thin boundary layer 25, 26. . Not only does this regularize the problem, but Ref. 25 has been shown to provide significantly better accuracy than the Myers boundary condition [27]. These regularizations have until now been restricted to the frequency-domain, and it is one purpose of this paper to present a time-domain finite-difference implementation of the modified boundary condition of Ref. 25 .

It should be noted that flow past a non-rigid boundary is often unstable, both in theory [28, 29] and in practice [30, 31], as can be appreciated by considering the flapping of a flag in the wind [32]. While the modified boundary conditions mentioned above remove the illposedness of arbitrarily fast exponential growth caused by overly simple modelling assumptions, they should therefore still be expected to result in most cases in a convectively unstable system [26, 33]. One other aim of this paper is therefore to describe general numerical difficulties that arise when simulating unstable linear systems. In particular, careful distinction is needed between genuine instabilities of the system being simulated and artificial instabilities introduced by the numerical discretization [19]. While there have been previous investigations of special cases, such as the careful numerical treatment of the continuous spectrum by Marx 24], here we propose for general simulations a general class of artificial instabilities caused by a combination of instability of the underlying physical system and the finite resolution of the numerical discretization. These artificial instabilities are of a similar nature to those caused directly by under-resolution and aliasing [e.g. 14], but are distinct on two accounts: firstly, they can be prevented by filtering only at the impedance boundary rather than throughout the fluid; and secondly, under-resolution in space leads to well-resolved exponential growth in time, rather than leading to dispersive and under-resolved temporal behaviour.

While we are concerned here with only inviscid flows, the techniques described here will be equally applicable to modified boundary conditions that incorporate viscosity [34 36], provided the modified boundary conditions remain well-posed. It is worth noting that viscosity by itself does not regularize the illposedness due to the assumption of an infinitely thin boundary layer [35], but viscosity is likely to be important for accuracy in certain situations [37, 38], and for stabilizing the well-posed inviscid instabilities [36].

In order to verify that the numerical scheme developed here accurately reproduces the correct results and stability of the underlying equations, we will here consider a simple situation of a time-harmonic line source in a uniform flow past an acoustic liner which admits an analytic solution [39]. Following a description of the general method in $\$ 2$, this simple situation is described in $\$ 3$, together with the numerical scheme used and a comparison of numerical results with the analytic solution. A discussion of the important subtleties of this numerical scheme owing to the convective instability of the underlying equations is given in 4 , in light of which the design and optimization of several boundary condition filters is described in $\$ 5$. The conclusion in $\$[$ also discusses possible future extensions of this work, including its application to other numerical schemes (such as finite elements) and the inclusion of viscosity. 


\section{Mathematical formulation}

We consider a fluid with velocity $\boldsymbol{u}$, pressure $p$ and density $\rho$. Neglecting viscosity, the governing equations are the Euler equations, given in appendix $\mathrm{A}$. We write the time-independent mean flow with a subscript zero and the small time-dependent perturbation with a prime, so that $\rho^{\prime}=\rho-\rho_{0}$ is the small time-dependent density perturbation and $(\rho \boldsymbol{u})^{\prime}=\rho \boldsymbol{u}-\rho_{0} \boldsymbol{u}_{0}$ is the small time-dependent momentum perturbation. The small perturbations are therefore governed by the Linearized Euler Equations (LEE), also given in appendix $\mathrm{A}$

\subsection{The impedance boundary}

We consider a flat impedance boundary, with coordinates chosen such that the boundary is at $y=0$ with the fluid in $y<0$ and the mean flow in the $\boldsymbol{e}_{x}$ direction. We assume the normal velocity of the fluid at the surface $v_{s}$ may be modelled by a linear time-evolution equation forced by the surface pressure perturbation $p^{\prime}$, and we denote this model as $\mathcal{B}\left(p^{\prime}\right)$. For example, a mass-spring-damper boundary has a normal displacement $\xi$ satisfying

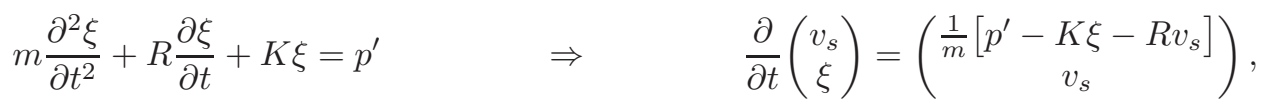

where $m, K$ and $R$ are the mass, spring and damping coefficients respectively. By assuming a time dependence $\exp \{\mathrm{i} \omega t\}$ to $\xi$ and $v_{s}$, equation (2) gives the frequency-domain impedance $Z(\omega)=R+$ $\mathrm{i}(m \omega-K / \omega)$. The reverse is also possible, at least for locally-reacting impedances: given a locallyreacting frequency-domain impedance $Z(\omega)$ such that the Fourier transforms $\hat{v}_{s}$ and $\hat{p}$ of $v_{s}$ and $p^{\prime}$ are related by $\hat{v}_{s}=\hat{p} / Z$, the convolution theorem gives

$$
v_{s}=\int_{-\infty}^{\infty} Y(\tau) p^{\prime}(t-\tau) \mathrm{d} \tau \quad \text { where } \quad Y(t)=\frac{1}{2 \pi} \int_{-\infty}^{\infty} Z(\omega)^{-1} \mathrm{e}^{\mathrm{i} \omega t} \mathrm{~d} \omega,
$$

and a causal boundary should satisfy $Y(\tau)=0$ for $\tau<0$, one of Rienstra's criteria for an admissible impedance boundary [5]. While the expression (3) is often not convenient computationally, other forms for $\mathcal{B}\left(p^{\prime}\right)$ are often possible that are significantly more convenient, with one example being the massspring-damper impedance given above in (2). Another example is the extended Helmholtz resonator model, which may be efficiently expressed using a $z$-transform [5] giving a result suitable for numerical use. Note, however, that $v_{s}$ is different from $v^{\prime}$ at $y=0$, and so a boundary condition linking $v_{s}$ and $v^{\prime}$ is needed.

\subsection{The Myers boundary condition}

In the frequency domain we write $v^{\prime}(x, y, z, t)=\hat{v}(y) \exp \{\mathrm{i} \omega t-\mathrm{i} k x-\mathrm{i} \ell z\}$, and similarly for the other variables. The Myers boundary condition (1) formulated in the frequency domain states that

$$
\mathrm{i} \omega Z \hat{v}=\mathrm{i}\left(\omega-u_{0} k\right) \hat{p} \quad \text { at } \quad y=0 .
$$

Noting that $\hat{p} / Z=\hat{v}_{s}$, in the time domain this boundary condition becomes

$$
\frac{\partial v^{\prime}}{\partial t}=\frac{\partial v_{s}}{\partial t}+u_{0} \frac{\partial v_{s}}{\partial x} \quad \text { or, more generally, } \quad \frac{\partial v^{\prime}}{\partial t}=\left(\frac{\partial}{\partial t}+\boldsymbol{u}_{0} \cdot \boldsymbol{\nabla}\right) v_{s},
$$

where $v_{s}$ is given by the time-domain boundary model $\mathcal{B}\left(p^{\prime}\right)$.

\subsection{A modified boundary condition accounting for a thin boundary layer}

Incorporating a thin boundary layer into the boundary condition, the modified frequency-domain boundary condition of Ref. 25 is given by

$$
\left[\mathrm{i} \omega Z+\rho_{0}\left(\omega-u_{0} k\right)^{2} \delta I_{0}\right] \hat{v}=\left[\mathrm{i}\left(\omega-u_{0} k\right)-\mathrm{i} \omega Z \delta I_{1} \frac{k^{2}+\ell^{2}}{\mathrm{i}\left(\omega-u_{0} k\right) \rho_{0}}\right] \hat{p},
$$


where $\delta I_{0}$ and $\delta I_{1}$ are small correction terms to (4) accounting for the presence of the thin boundary layer. If we assume a boundary layer of width $\delta$ with a linear velocity variation and a uniform density, then

$$
\left(\omega-u_{0} k\right)^{2} \delta I_{0}=-\omega k u_{0} \delta+\frac{2}{3} k^{2} u_{0}^{2} \delta, \quad \text { and } \quad \delta I_{1}=\delta u_{0} k / \omega .
$$

In the derivation of Ref. 25, the flow outside the boundary layer was assumed constant, and so the linearized momentum equation in the direction of the flow meant the identity $\mathrm{i} \rho_{0}\left(\omega-u_{0} k\right) \hat{u}-\mathrm{i} k \hat{p}=0$ held outside the boundary layer. It is therefore consistent with the assumptions of Ref. 25 to use this identity, together with (7), to transform (6) into

$$
\mathrm{i} \omega \hat{v}-\delta \rho_{0} u_{0} k\left(\omega-\frac{2}{3} u_{0} k\right) \hat{\nu}=\mathrm{i}\left(\omega-u_{0} k\right) \hat{v}_{s}-\delta u_{0}\left(k^{2}+\ell^{2}\right) \hat{u},
$$

where $\hat{v}_{s}=\hat{p} / Z$ and $\hat{\nu}=\hat{v} / Z$. This may be interpreted in the time domain as

$$
\frac{\partial v^{\prime}}{\partial t}=\left(\frac{\partial}{\partial t}+\boldsymbol{u}_{0} \cdot \nabla\right) v_{s}+\delta \boldsymbol{u}_{0} \cdot\left(\nabla_{\perp}^{2} \boldsymbol{u}^{\prime}\right)+\delta \rho_{0} \boldsymbol{u}_{0} \cdot \nabla\left(\frac{\partial}{\partial t}+\frac{2}{3} \boldsymbol{u}_{0} \cdot \nabla\right) \nu,
$$

where $\nabla_{\perp}$ is the gradient operator normal to the surface, $v_{s}$ is given by the time-domain boundary model $\mathcal{B}\left(p^{\prime}\right)$ and $\nu$ is given by $\mathcal{B}\left(v^{\prime}\right)$; i.e. the same time-domain boundary model $\mathcal{B}$ as for $v_{s}$ but forced by $v^{\prime}$ rather than $p^{\prime}$. For example, for the mass-spring-damper impedance,

$$
\begin{array}{ll}
\frac{\partial v_{s}}{\partial t}=\frac{1}{m}\left[p^{\prime}-K \xi-R v_{s}\right], & \frac{\partial \xi}{\partial t}=v_{s} \\
\frac{\partial \nu}{\partial t}=\frac{1}{m}\left[v^{\prime}-K \eta-R \nu\right], & \frac{\partial \eta}{\partial t}=\nu .
\end{array}
$$

The time-domain boundary condition (9) is one of the main results of this paper.

\subsection{Using characteristics to apply the boundary condition}

Away from the boundary, the time-evolution of the perturbation is given by the linearized Euler equations. At the impedance boundary, both the Myers boundary condition (5) and the modified boundary condition (9) specify the value of $\partial v^{\prime} / \partial t$ at the boundary, thus specifying a different time evolution for $v^{\prime}$. Neither boundary condition puts any restrictions on the time derivatives of any other perturbed variable. One obvious possibility would therefore be to evolve these other variables at the boundary using the same governing linearized Euler equations as within the fluid. We call this here the direct method of applying the boundary conditions. That is, using the direct method, the time derivatives of all quantities are calculated everywhere in the fluid using the linearized Euler equations, except that the boundary condition is used to give the time derivative of $v^{\prime}$ along the boundary.

As the direct method will be seen later to perform poorly, we now describe an alternative method, here termed the characteristic method. In this method, the time derivatives of all quantities are first calculated using the linearized Euler equations. These time derivatives are then modified at the boundary in order that the boundary condition be satisfied. Instead of just modifying the time derivative of $v^{\prime}$, however, we instead modify only the amplitude of the incoming characteristic, leaving the other characteristics unaltered. Details of this calculation are given in appendix A.1. The end result for a perfect gas with a homentropic mean flow is that, if $\dot{\boldsymbol{q}}=\frac{\partial}{\partial t}\left(\rho^{\prime}, u^{\prime}, v^{\prime}, w^{\prime}, p^{\prime}\right)$ as calculated by the linearized Euler equations at the boundary, then the vector of modified time derivatives $\dot{\boldsymbol{q}}^{\text {bc }}$ at the boundary is given by

$$
\dot{\boldsymbol{q}}^{\mathrm{bc}}=\dot{\boldsymbol{q}}-\left[\frac{\dot{q}_{5}}{2 \rho_{0} c_{0}^{2}}-\frac{\dot{q}_{3}}{2 c_{0}}-(1-\mathcal{F}) \theta\right]\left(\begin{array}{c}
\rho_{0} \\
0 \\
-c_{0} \\
0 \\
\rho_{0} c_{0}^{2}
\end{array}\right) \quad \text { where } \quad \theta=\frac{\dot{q}_{5}}{2 \rho_{0} c_{0}^{2}}+\frac{\dot{q}_{3}}{2 c_{0}}-\frac{1}{c_{0}} \frac{\partial v^{\prime}}{\partial t},
$$

where $\theta$ is the amplitude of the incoming characteristic, $c_{0}=\sqrt{\gamma p_{0} / \rho_{0}}$ is the local sound speed, $\dot{q}_{3}$ and $\dot{q}_{5}$ are the third and fifth components of the vector $\dot{\boldsymbol{q}}$, and $\mathcal{F}$ is an optional spatial filtering that will be discussed in detail in \$5. Equation (11) constitutes our time-domain characteristics-based impedance 


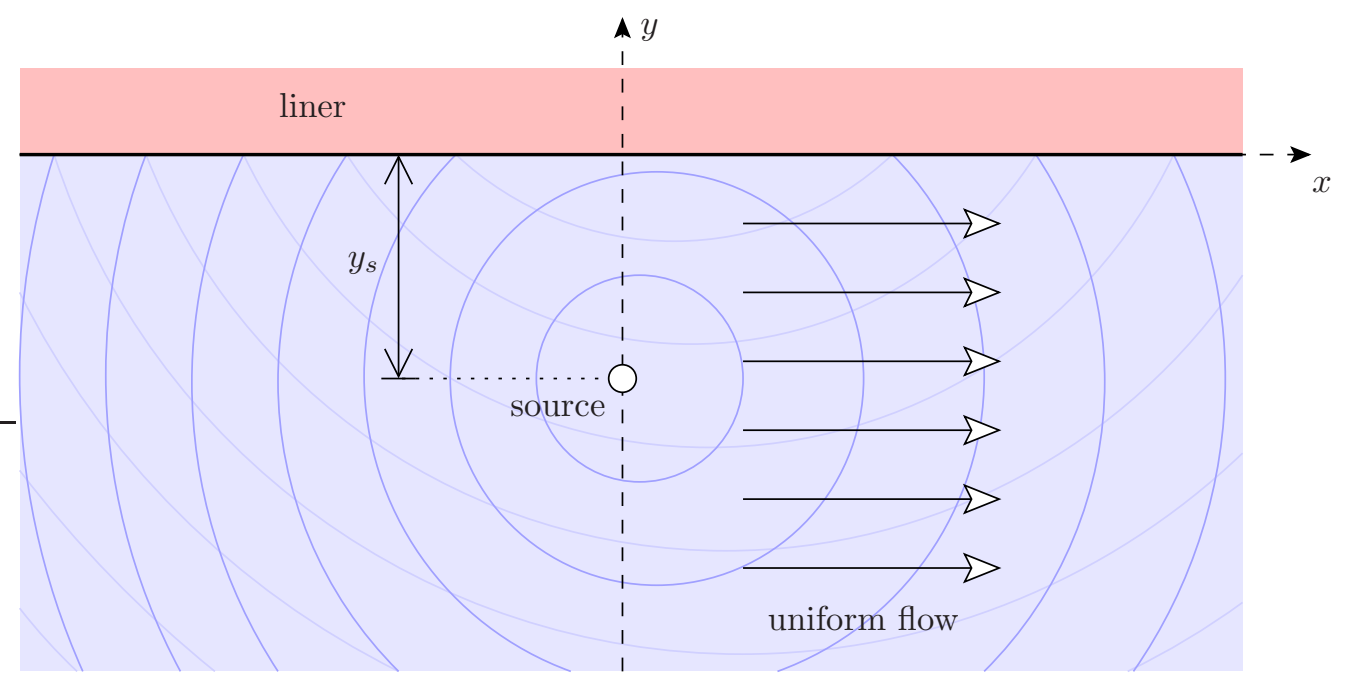

Figure 1: Diagram of the benchmark problem.

boundary condition, with $\partial v^{\prime} / \partial t$ on the right hand side of (11) given by either boundary condition (5) or (9). This may be contrasted against the direct method written in the same form,

$$
\dot{\boldsymbol{q}}^{\mathrm{bc}}=\dot{\boldsymbol{q}}-\left[\dot{q}_{3}-(1-\mathcal{F}) \frac{\partial v^{\prime}}{\partial t}\right]\left(\begin{array}{l}
0 \\
0 \\
1 \\
0 \\
0
\end{array}\right) .
$$

While (11) and (12) have been specialized to the case of a homentropic mean flow of a perfect gas, this is not necessary and the general result is given in appendix A.1

\section{Test case and numerical implementation}

In order to analyse the behaviour of the numerical implementation of the above boundary conditions, particularly with regard to stability, it is important to have a known correct solution to compare to. We achieve this here by considering as a test case a simple two-dimensional situation that has previously been investigated analytically [39], sketched in figure 1. A fluid with constant density $\rho_{0} \equiv 1$ and constant sound speed $c_{0} \equiv 1$ occupies $y<0$ and flows with uniform Mach number $M$ in the positive $x$-direction past a wall at $y=0$, with the Euler equations therefore implying the steady pressure $p_{0}$ is constant. A small oscillatory point mass source 11 of strength $Q^{\prime}=\delta(x) \delta\left(y+y_{s}\right) H(t) \sin (\omega t)$ generates small perturbations to this steady flow, and the wall at $y=0$ responds as a mass-spring-damper boundary governed by (2).

A time-domain finite-difference numerical simulation of this test case is performed using standard numerical techniques, with the exception of the numerical impedance boundary condition which is implemented using (11) and (9). Full details of the equations solved and numerical scheme used are given in appendix $\mathrm{B}$. The equations are solved on a truncated rectangular domain $-L^{-} \leq x \leq L^{+}$and $-H \leq y \leq 0$, with grid-points spaced equidistantly by $\Delta x$ and $\Delta y$ in the $x$ - and $y$-directions. Spatial derivatives are calculated using a seven-point fourth-order DRP scheme [11, 15] (with non-centered stencils used near the domain boundaries), and time marching is performed using a six-stage fourth-order Runge-Kutta time step [12] of overall length $\Delta t$. The CFL number $(1+M) \Delta t / \Delta x$ is chosen in the interval $[0.8,0.9]$. A seven-point selective spatial filter (a sixth order filter s7 described in section 5) is applied with small strength $S_{\mathrm{F}}$ throughout the domain at each time step to remove spurious shortwavelength components. The point source is implemented numerically as a Gaussian monopole of width

\footnotetext{
${ }^{1}$ by which we mean a point mass source in two dimensions, or equivalently, a line mass source along the third dimension in three dimensions.
} 


\begin{tabular}{rc|ccc} 
& & $\mathrm{A}$ & $\mathrm{B}$ & $\mathrm{C}$ \\
\hline Flow Mach number & $M$ & 0.5 & 0.5 & 0.4 \\
Source frequency & $\omega$ & 31 & 31 & 10 \\
Source position & $y_{s}$ & 0.3 & 0.3 & 0.3 \\
Boundary layer thickness & $\delta$ & 0.001 & 0.001 & 0.001 \\
Impedance mass & $m$ & $\mid$ & 0.01 & 0.08 \\
Impedance spring & $K$ & rigid & 10 & 6 \\
Impedance damping & $R$ & $\mid$ & 0.75 & 1.6 \\
Impedance resonant frequency & $\sqrt{K / m}$ & & 31.6 & 8.66 \\
Impedance damping factor & $R / \sqrt{4 K m}$ & & 1.19 & 1.15 \\
Grid 1 spacing (coarse) & $\Delta x=\Delta y$ & 0.01 & 0.01 & 0.03 \\
Grid 2 spacing & $\Delta x=\Delta y$ & 0.005 & 0.005 & 0.015 \\
Grid 3 spacing & $\Delta x=\Delta y$ & 0.0025 & 0.0025 & 0.003 \\
Grid 4 spacing (fine) & $\Delta x=\Delta y$ & 0.001 & 0.001 & 0.002
\end{tabular}

Table 1: Parameters used for the different test cases investigated here.

$w=\Delta x+\Delta y$. A PML nonreflecting boundary [40] is applied at the bottom of the domain, and periodic boundary conditions connect the upstream and downstream ends of the domain at $x=L^{ \pm}$. The impedance boundary condition is implemented by modifying the incoming characteristic at the boundary $y=0$, as described in (11), with the velocity derivative $\partial v^{\prime} / \partial t$ given by the modified boundary condition (9) and the mass-spring-damper modelled using (10); this requires storage of four extra quantities $\left(v_{s}, \xi, \nu\right.$ and $\left.\eta\right)$ at each location along the boundary. The same spatial and temporal stencils were used for the boundary conditions as were used for the LEE. The boundary filter $\mathcal{F}$ in (11) was chosen to be the fourth order $\mathrm{n} 7$ filter described in $\$ 5$, effects of using different filtering will also be discussed in $\$ 5$,

To validate the numerical implementation, and to investigate the effects of different implementations of the impedance boundary condition, numerical results are compared with two other solutions. The first is the analytic modal analysis and long-time limit of Ref. 39, calculated using the code in the supplementary material of that reference. Since this is an analytic solution of the LEE in an unbounded half-space, effects due to discretization and the finite computational domain size are absent in this solution. Since the analytic long-time solution is only valid in the long-time limit, it can only be compared to the numerical solution once the initial transients of the sudden start at $t=0$ have propagated away. The modal analysis of Ref. 39, and in particular the temporal stability analysis, can also be used to compare the most unstable modal content of the numerical simulation with that predicted analytically.

As a second comparison tool, the discretized dispersion analysis (DDA) of Ref. 19 is also performed. This analysis assumes a spatial and temporal dependence $\exp \{\mathrm{i} \omega t-\mathrm{i} k x\}$ but otherwise exactly reproduces the numerical method described above; that is, the DDA includes the discretization, the DRP scheme, the time-stepping, the numerical implementation of the boundary condition, the PML at the lower boundary, and the numerical filtering. This results in a linear eigenvalue problem to be solved for any given spatial wavenumber $k$, with the eigenvalue $\omega$ giving the time evolution and the eigenvectors giving the spatial mode shapes. For further details the reader is referred to Ref. 19. Once the DDA is validated against the full numerical time-domain solution (and the agreement will be seen to be excellent), the DDA can be used to investigate the effects of varying parameters, introducing or removing damping, and changing the way the boundary condition is discretized and implemented, without the noise inherent in post-processing the output of the full time-domain solution.

\subsection{Numerical results}

Several sets of parameters are considered for the situation described above. Three test cases were investigated, labelled $\mathrm{A}-\mathrm{C}$, with each test case investigated at four resolutions. The coarsest resolution was sufficient to resolve the acoustic waves at 10 points per wavelength, and the finest resolution was sufficient to resolve the unstable surface waves at at least 10 points per wavelength. A summary of important parameters for these test cases is given in table 1] with a full list of parameters given in appendix B 

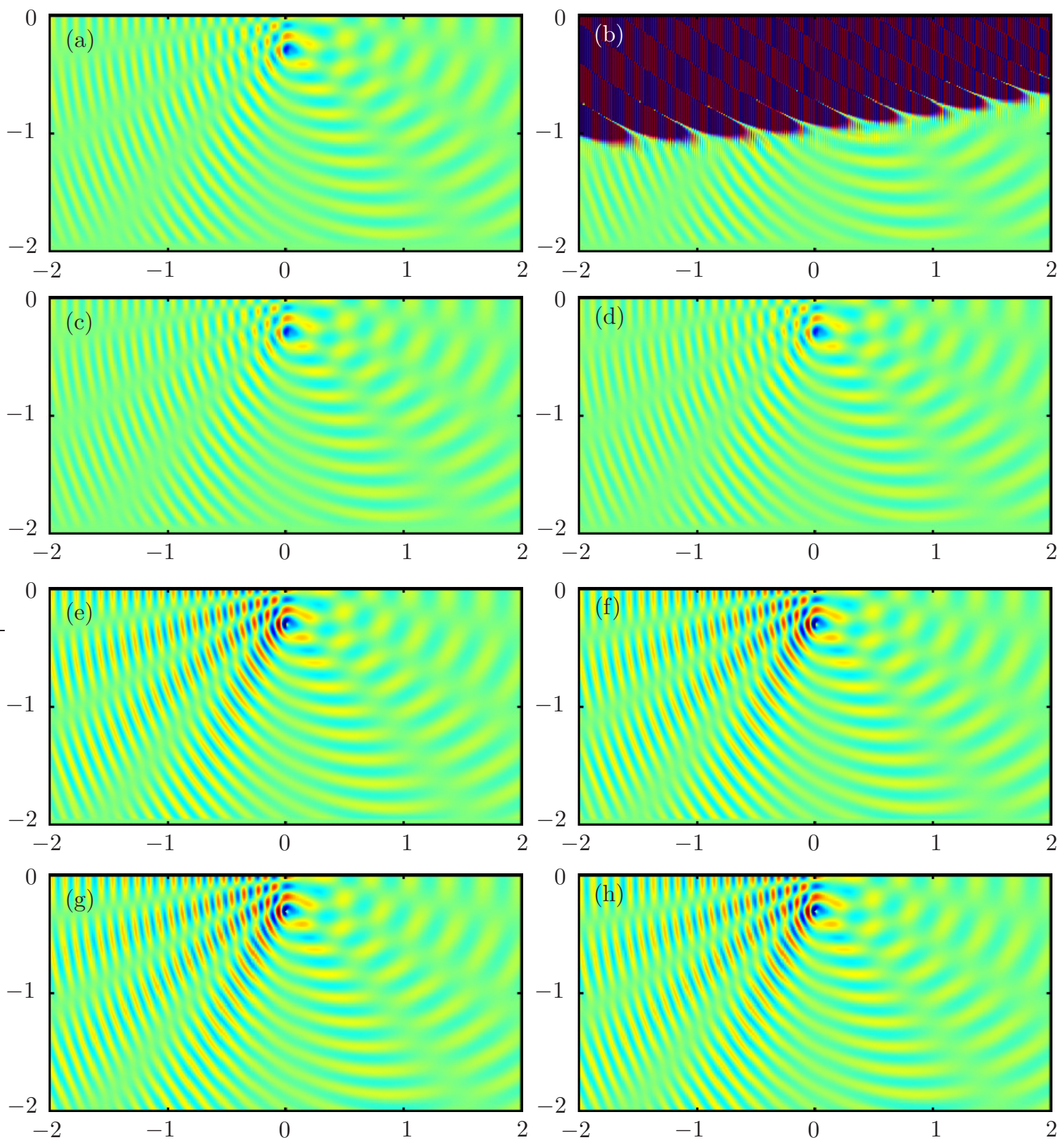

Figure 2: Pressure (red positive, blue negative) at time $t=4.62$ for case A (table 1), having a rigid wall with impedance $Z=\infty$ along $y=0$. (a), (c), (e) and (g) have no boundary layer $(\delta=0)$, while (b), (d), (f) and (h) have a boundary layer of thickness $\delta=10^{-3}$. (a) and (b) perform no boundary filtering, while (c)-(f) apply the $\mathrm{n} 7$ filter (section 5) to the incoming characteristic. (a)-(d) use the course grid 1 with $\Delta x=10^{-2}$, while (e) and (f) use the fine grid 4 with $\Delta x=10^{-3}$. (g) and (h) are the analytic results of Ref. 39 .

\subsubsection{Case A: rigid walls $(Z=\infty)$}

Figure 2 shows a snapshot of the hard-walled test case A at time $t=4.62$, sufficient time that the initial transients from the sudden start at $t=0$ have propagated away. The left hand column uses the Myers boundary condition (5), while the right hand column uses the modified boundary condition (9) with a boundary layer of width $\delta=10^{-3}$. Subfigures (a)-(d) use the coarsest grid with 10 points per acoustic wavelength. An instability is seen in subfigure (b) since neither (a) nor (b) use filtering in their boundary conditions, but (c) and (d) show that introducing filtering removes this instability without affecting the accuracy of the acoustic solution. This artificial numerical instability will be investigated further in the following sections concerning non-rigid boundaries. Subfigures (e) and (f) are for a ten times higher grid resolution; the only difference visible between (c)-(d) and (e)-(f) are a stronger 
(a)

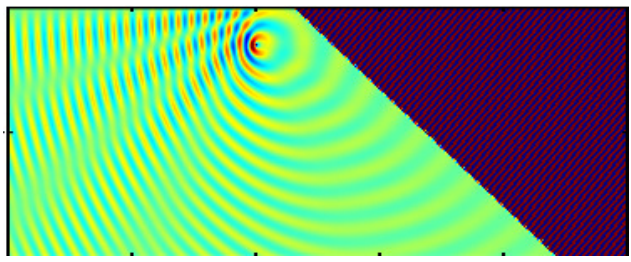

(c)

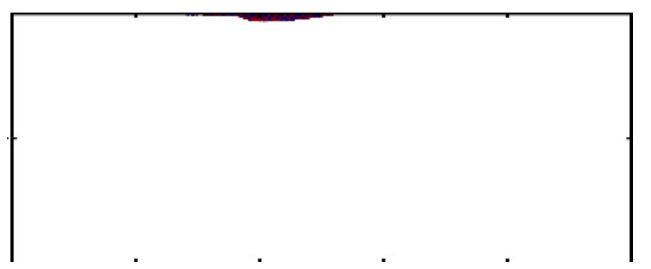

(e)

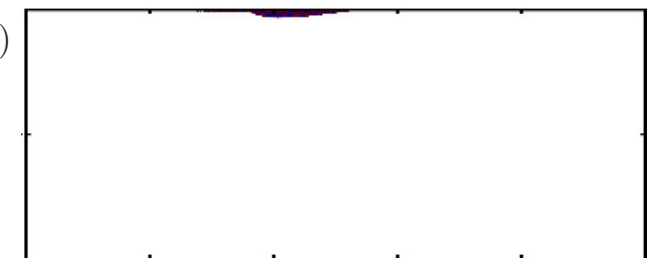

(g)

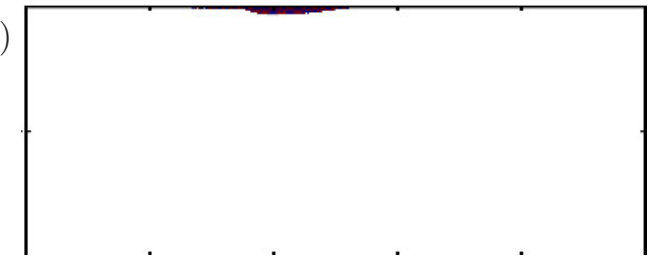

(i)

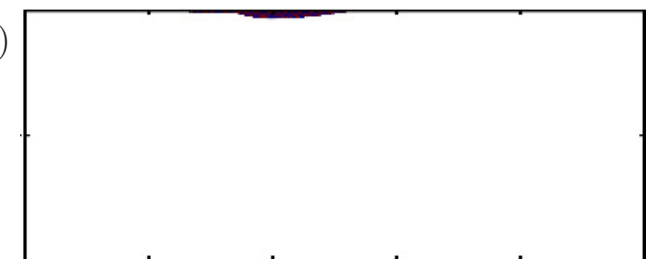

(b)

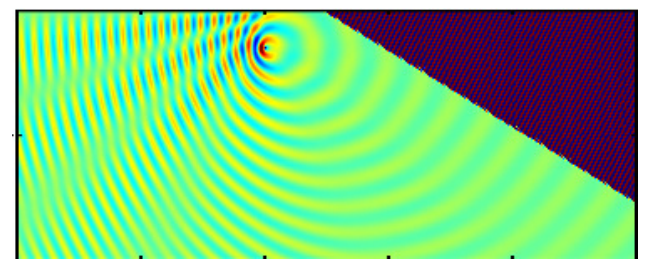

(d)

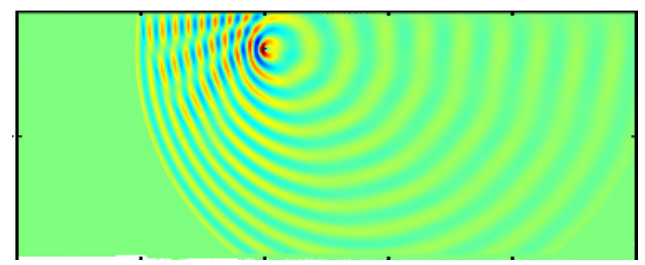

(f)

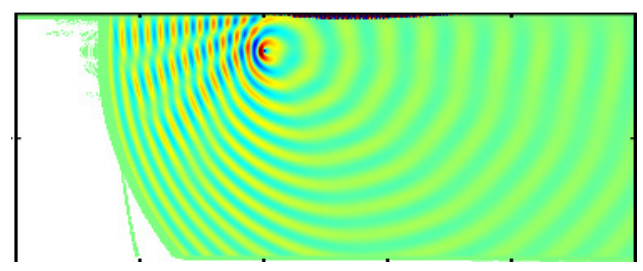

(h)

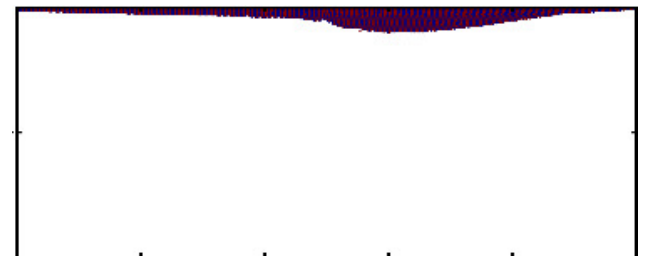

(j)

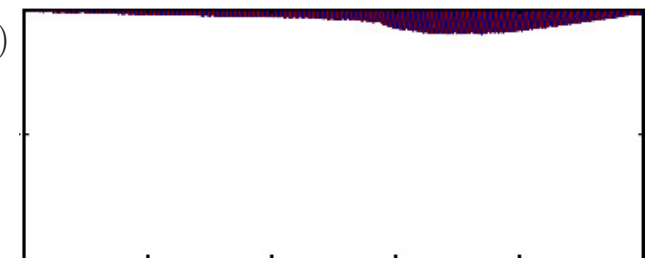

Figure 3: Plots of pressure for case B for $\delta=0$ (a,c,e,g,i) and $\delta=10^{-3}$ (b,d,f,h,j) calculated numerically at times $t=2.1(\mathrm{c}, \mathrm{d}), t=2.5(\mathrm{e}, \mathrm{f}), t=4.2(\mathrm{~g}, \mathrm{~h})$ and $t=6.0(\mathrm{i}, \mathrm{j})$. For comparison, $(\mathrm{a}, \mathrm{b})$ are the analytic results from Ref. 39. Red is positive pressure, blue is negative pressure. For the numerical results, white regions are regions where the amplitude is less than $2 \times 10^{-16}$ times the maximum pressure and so are within the numerical double-precision noise.

upstream propagation in the latter, caused by the Gaussian source being more compact in (e)-(f) and therefore better approximating a true point source. Indeed, the comparison between (e)-(f) and the analytic results in $(\mathrm{g})-(\mathrm{h})$ shows no discernible difference, suggesting the correctness of the numerical scheme once the point source is adequately resolved. The only effect of the thin boundary layer is to slightly alter the angle of the lobes radiating in the upstream direction; such a small effect is due to the wall being entirely rigid in this case.

\subsubsection{Case B: lined walls}

Figure 3 plots several snapshot of the lined-walled test case B at various times for both the Myers boundary condition (5) in the left-hand-column and the modified boundary condition (9) in the righthand-column. Subfigures (a)-(b) show the analytic long-time result, and clearly show a convective instability. Since this instability grows exponentially in space, and since the numerical simulations in (c)-(j) use double precision floating point calculations with a rounding error of approximately $2 \times 10^{-16}$, for these numerical simulations only regions with an amplitude at least $2 \times 10^{-16}$ times the maximum amplitude are plotted, since other regions will contain only numerical noise. For the Myers boundary 
(a) $\delta=10^{-3}, U=0.25, G=23.5$

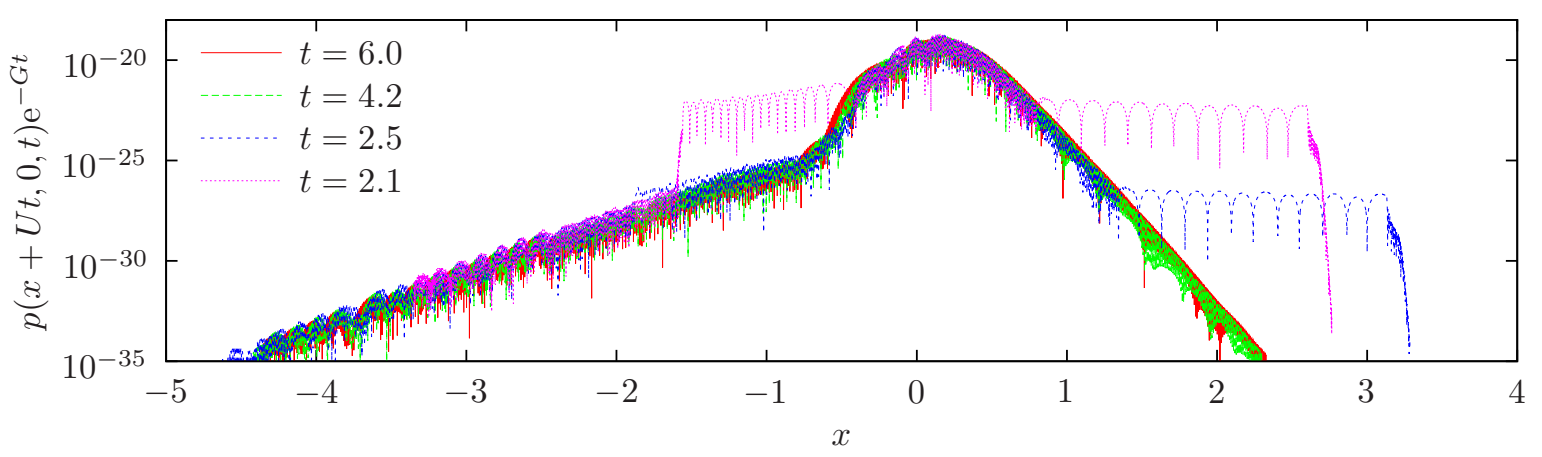

(b) $\delta=0, U=-0.01, G=121$

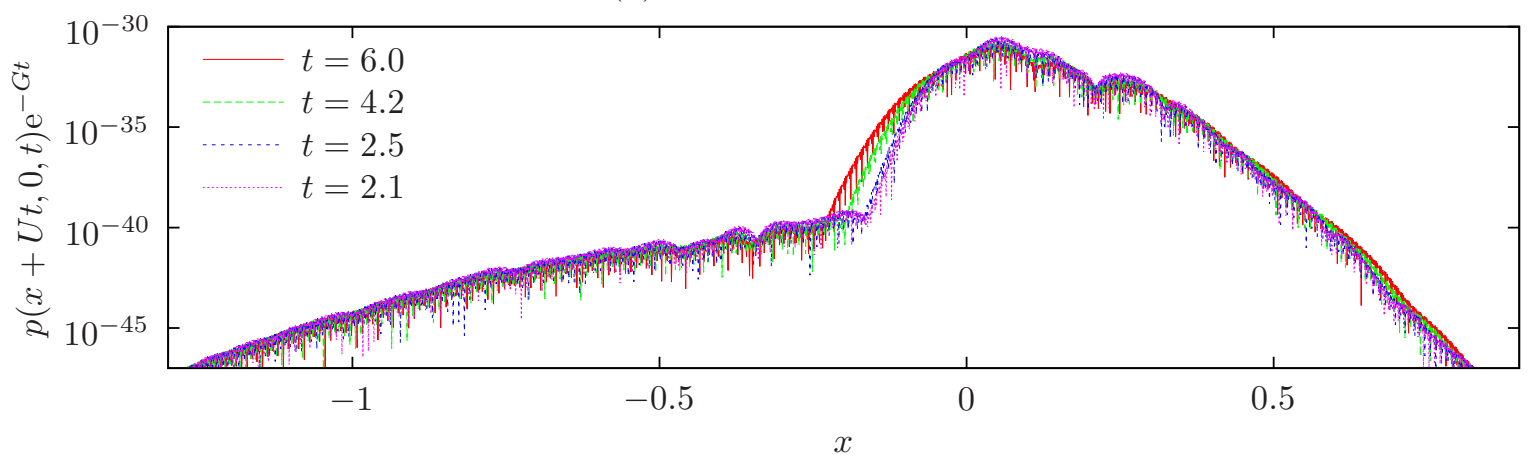

Figure 4: Plots of $p(x+U t, 0, t) \mathrm{e}^{-G t}$, where $p(x, 0, t)$ is the numerically calculated pressure along the lining $y=0$ for grid 4 of case B (table 1). (a) includes the boundary layer with incoming characteristic filtered using the $\mathrm{n} 7$ filter (section 5). (b) ignores the boundary layer but still filters the incoming characteristic with filter $\mathrm{n} 7$. Note that (a) and (b) are plotted using different scales.

condition an absolute instability is seen which grows in time at all spatial locations, while for the $\delta=10^{-3}$ case a more slowly-growing convective instability is present that propagates downstream.

In order to better quantify the instabilities seen in figure 3 , figure 4 plots the pressure amplitude along the liner at $y=0$ on a $\log$ scale for each of the times plotted in figure 3 The pressure at each time is multiplied by $\mathrm{e}^{-G t}$ and translated by $-U t$, with $G$ and $U$ chosen so as to collapse the instability onto a single curve as far as possible. Figure 4(a) for the modified boundary condition shows a wave packet convecting at half the mean flow velocity (i.e. $U=0.25$ ) and growing with a growth rate $G=23.5$. At the earlier times $t=2.1$ and $t=2.5$ the acoustic signal is visible away from the convecting wave packet, but by $t=4.2$ it has been obliterated by the growth of the wave packet. By comparison, figure 4(b) shows the same results for the Myers boundary condition, and clearly shows a non-convective wave packet $(U=-0.01)$ growing with a far higher growth rate $G=121$.

While the instability in figure $3(\mathrm{~h}, \mathrm{j})$ might at first sight seem similar to the instability in figure $2(\mathrm{~b})$ that was filtered out, in fact the instability in this case is genuine and is predicted by the analytic results of Ref. 39. By using the surface wave dispersion relation of Ref. 33 we may search for surface modes with $\exp \{\mathrm{i} \omega t-\mathrm{i} k x\}$ dependence such that the group velocity $c_{g}=\mathrm{d} \omega / \mathrm{d} k$ is real. An observer moving with such a group velocity would therefore observe a perturbation with dependence $\exp \left\{\mathrm{i}\left(\omega-k c_{g}\right) t\right\}$. Figure 5 plots this growth rate $-\operatorname{Im}\left(\omega-k c_{g}\right)$ as a function of the group velocity $c_{g}$, and clearly demonstrates that the most unstable wave packets will be seen with a group velocity of $U=0.248$ and a growth rate of $G=23.48$, in agreement with figure 4 (a). The numerical instability seen in this case may therefore be attributed to being an accurate simulation of a genuine instability of the underlying equations, rather than an artificial instability that is an artifact of the numerical scheme used. Since the Myers boundary condition leads to an ill-posed mathematical problem, it is not possible to perform a comparable analysis for the $\delta=0$ case; see $\$ 3.3$ or refer to Ref. 20 for further details. 


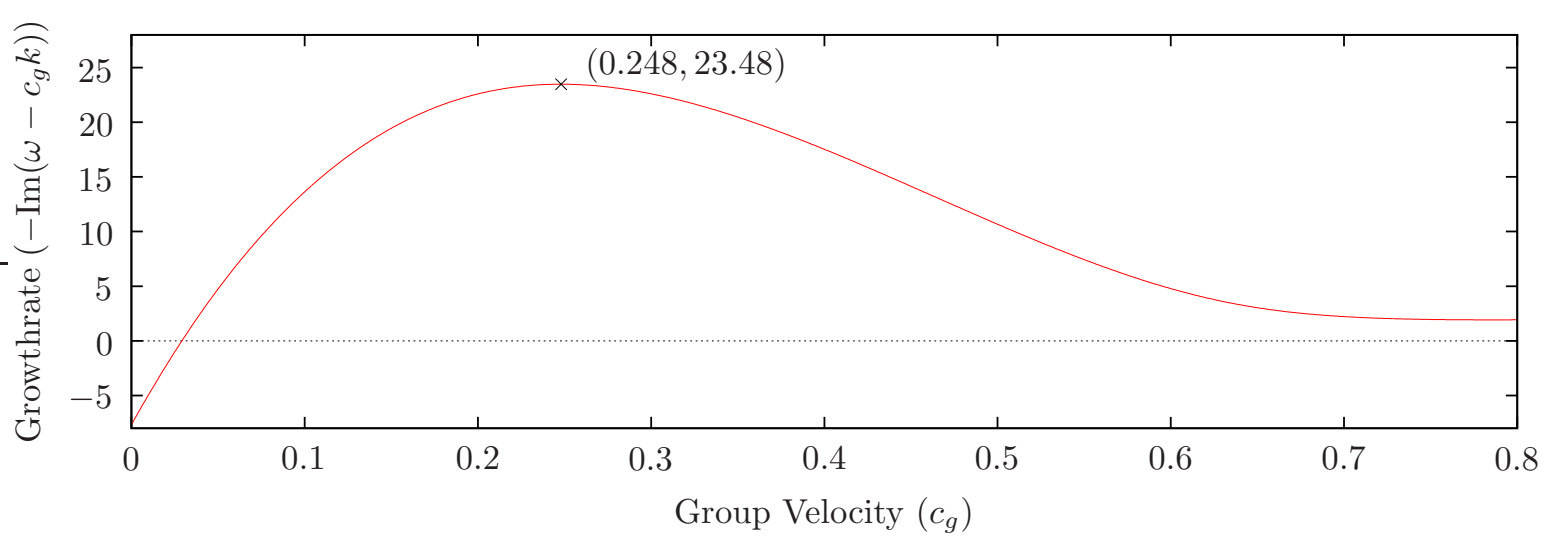

Figure 5: For case B, plot of the asymptotic growth rate $\left(-\operatorname{Im}\left(\omega-c_{g} k\right)\right)$ when travelling with velocity $x=c_{g} t$ predicted by the analytic results of Ref. 39 .

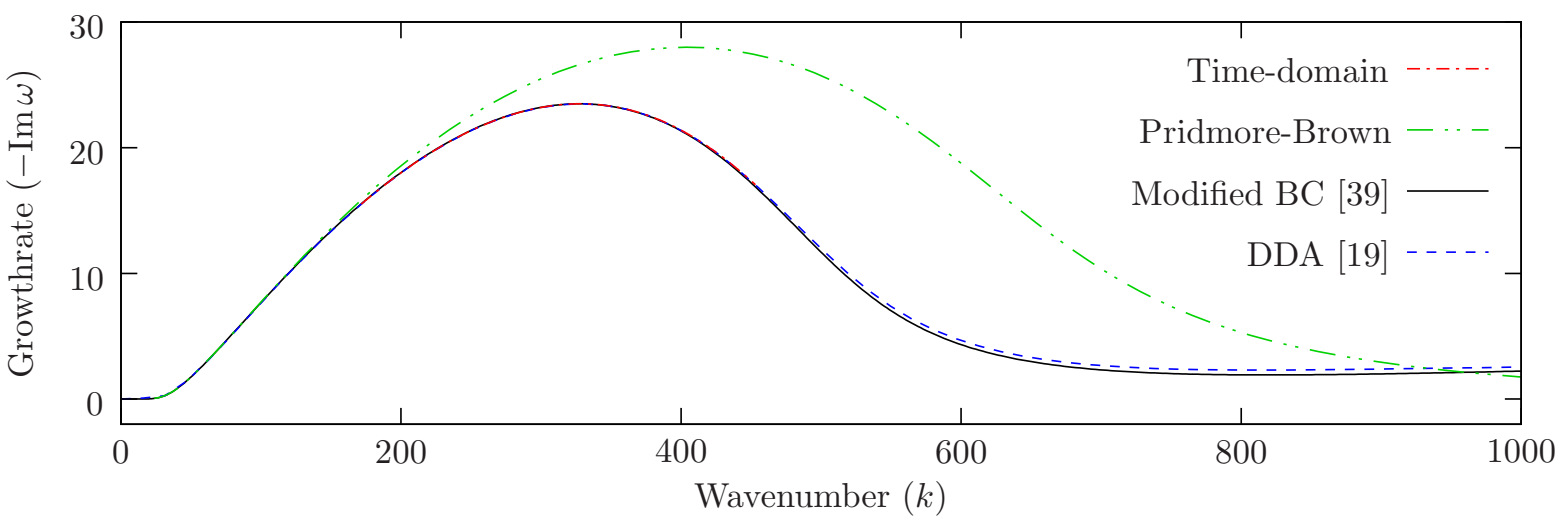

Figure 6: Exponential growth rate as a function of wavenumber for case B, grid 4. The time-domain computational result is obtained from a discrete Fourier transform of the growth between times $t=3.2$ and $t=4.5$. Also plotted are growth rates given by the discrete dispersion analysis [19], the continuous analytic result using the modified boundary condition [39], and the dispersion analysis from solving the Pridmore-Brown equation resolving the boundary layer.

\subsubsection{Case C}

The results for case $\mathrm{C}$ are similar to case B, and the conclusions are the same. Results are therefore omitted here for brevity.

\subsection{Temporal growth rates}

We next compare the temporal growth rate $-\operatorname{Im} \omega$ extracted from the time-domain simulation with those expected from both the analytic theory [39] and from the discrete dispersion analysis (DDA) of Ref. 19, for different spatial Fourier components $k$. For the time-domain simulation, the growth rate is obtained by performing a discrete Fourier transform on the pressure amplitude along the lining at $y=0$ and taking the ratio of the magnitudes of the Fourier components at times $t=4.5$ and $t=3.2$. Due to the rounding errors of double precision floating point numbers mentioned above, only Fourier components of sufficiently large magnitude give meaningful growth rates. Figure 6 plots the growth rates found in the time-domain simulation of case B at the finest grid resolution, and compares those with the prediction from the DDA; excellent agreement is seen, giving confidence in the DDA predictions even at smaller growth rates which are too small to be seen in the time-domain simulations due to numerical rounding errors. Excellent agreement is also seen between the analytic and discrete solutions in figure 6 . However, the modified boundary condition is only an approximation of perturbations to an inviscid sheared flow governed by the Pridmore-Brown equation [41], and the approximation is only valid for small $\delta$ and $k \ll 1 / \delta$. The discrepancy between resolving the boundary layer using the Pridmore-Brown equation and approximating the boundary layer using the modified boundary condition can be seen in 

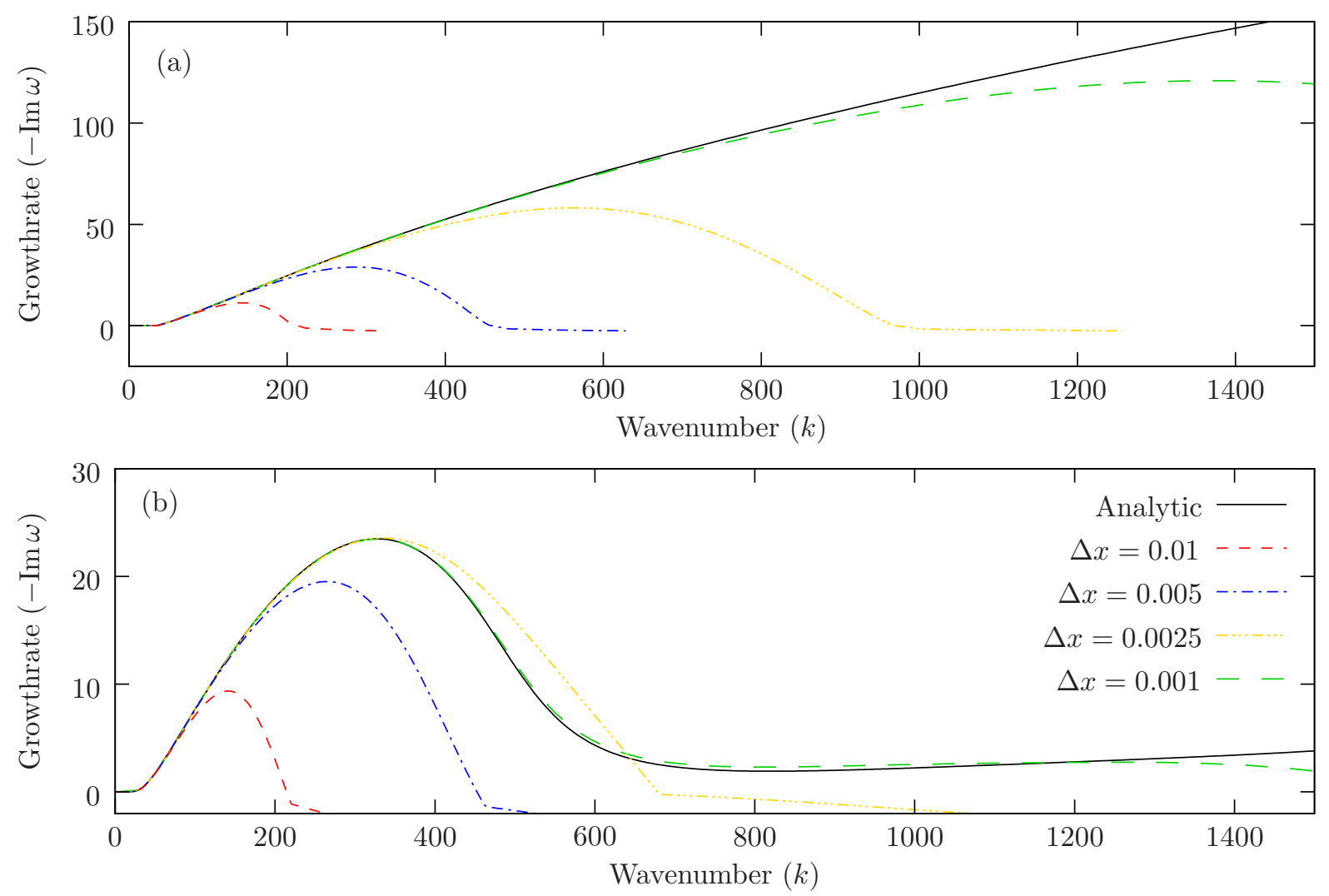

Figure 7: For case B, plots of the growth rate $(-\operatorname{Im} \omega)$ predicted by Ref. 19 against wavenumber $k$ for various grid resolutions, together with the analytic solution using either the Myers boundary condition [8] or the modified boundary condition from Ref. 39 .

figure 6 despite the very small value of $\delta=10^{-3}$ used here; time-domain simulations therefore need only simulate the underlying modified boundary condition as accurately as the modified boundary condition reproduces the Pridmore-Brown boundary-layer-resolved results.

Excellent agreement was also seen between growth rates extracted from time-domain simulations and growth rates predicted by the DDA in numerous other test cases not plotted here, including those for which there is a significant deviation from the analytic results due to under-resolution and other effects of discretization discussed below; a selection of these are given in the supplementary material. We can therefore be confident that the DDA results represent the behaviour of the time-domain simulations.

\subsection{Convergence and wellposedness}

The primary effect of including the boundary layer within the modified boundary condition (9) rather than using the simpler Myers boundary condition (5) is to make the underlying mathematical problem well-posed (see Ref. 25). The numerical impact of this is that numerical simulations will converge as the computational grid is refined, whereas for the illposed problem yet finer grids will lead to yet-quicker instability growth which remains poorly resolved spatially. To illustrate this, figure 7 plots the temporal growth rate for case B for several spatial resolutions for both the Myers (a) and modified (b) boundary conditions. The analytic results (corresponding to an infinitely fine grid spacing) are also plotted. In both cases, the time-domain numerics accurately reflect the analytic solution at well resolved length scales (small $k$ ) before failing to correctly resolve the instability at shorter length scales (larger $k$ ) as first the accuracy of the numerical derivative fails and subsequently the Nyquist frequency $k=\pi / \Delta x$ is reached. Figure 7(a) for the Myers boundary condition shows that, as the grid is refined, the point of maximum instability grows and shifts to larger wavenumbers, leading to ever faster growing instability at barely resolved spatial length scales; the is due to the underlying mathematical model being illposed and allowing arbitrarily high growth rates for arbitrarily large $k$. In contrast, figure 7 (b) for the modified boundary condition shows that, as the grid is refined, the time-domain simulations converge to the 


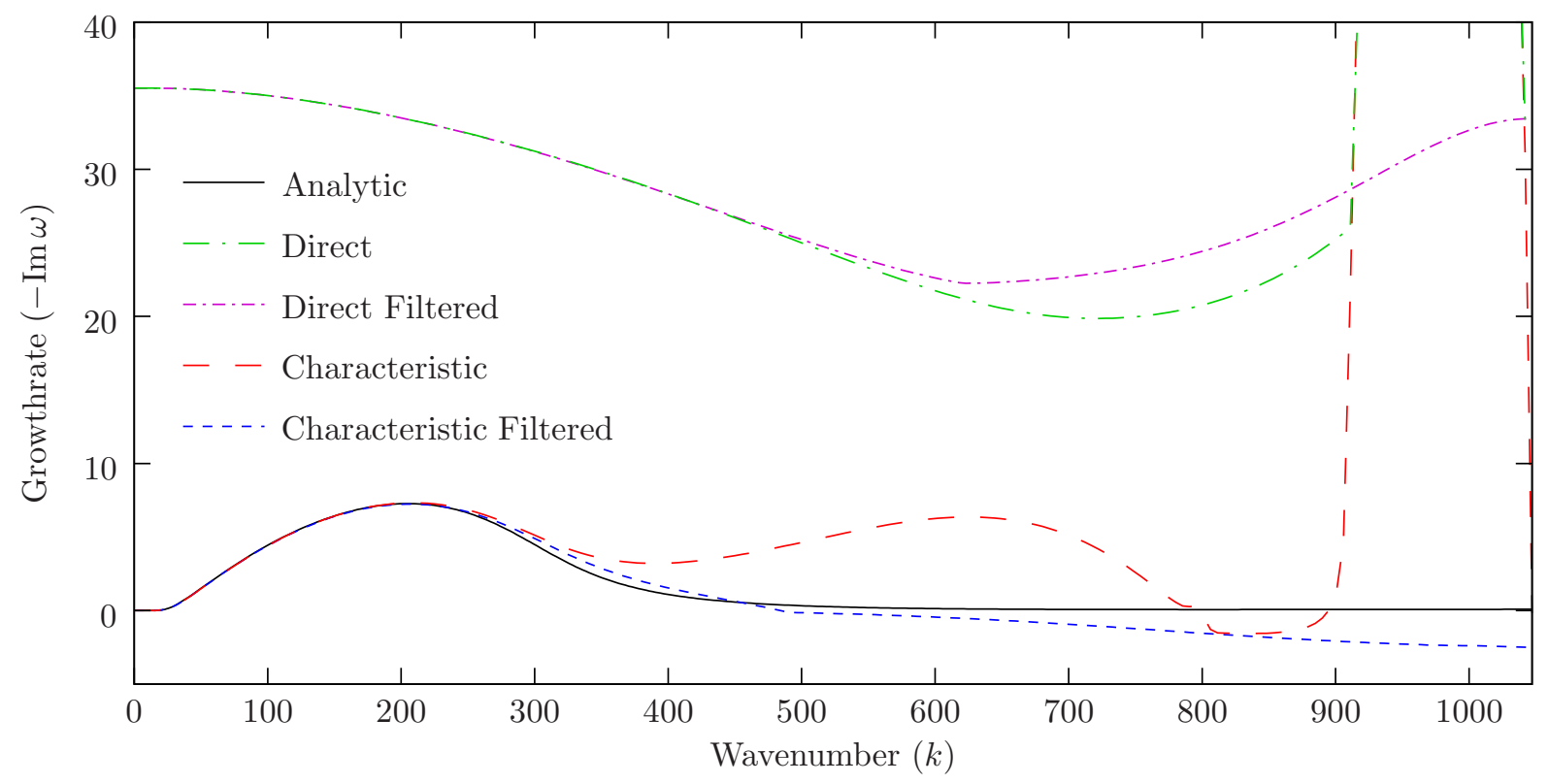

Figure 8: Growth rate against wavenumber for grid 3 of case $\mathrm{C}$ for various implementations of the boundary condition. The two curves labelled "direct" apply the boundary condition directly using (12), while the two curves labelled "characteristic" modify the incoming characteristic using (11). In the filtered cases, the n7 filter described in $\$ 5$ is used.

theoretical result which has a finite maximum growth rate (in this case, $-\operatorname{Im}(\omega) \approx 23.5$ for $k \approx 328$, of the same order of magnitude as the theoretical order-of-magnitude estimate $k \approx\left(1-M^{2}\right)^{1 / 6} m^{-1 / 3} \delta^{-2 / 3} \approx$ 442 from Ref. 33).

\section{Distinguishing real and artificial numerical instabilities}

In addition to formulating the modified boundary condition of Ref. 25 in the time domain, leading to equation (9), the implementation presented above applies this boundary condition using the incoming characteristic (11) rather than directly applying the boundary condition (12). A spatial filter $1-\mathcal{F}$ is also applied to the incoming characteristic amplitude $\theta$. In this section we discuss why these details are important, and what the consequences are of doing otherwise. Details of the filter $\mathcal{F}$ follow in section 5 ,

\subsection{Importance of using characteristic boundary conditions}

Figure 8 plots the results of the DDA 2 for grid 3 of case $\mathrm{C}$ for various methods of implementing the boundary condition (9). With or without filtering, directly applying the boundary condition using (12) is seen to lead to an unstable and inaccurate result. This may be because directly applying the boundary condition violates the causal behaviour that is preserved by using characteristics, where only characteristics propagating into the domain may be affected by the presence of the boundary. Owing to these problems with directly applying the boundary conditions, we will only consider a characteristics-based boundary condition given by (11) in what follows.

With the incoming characteristic filtered, figure 8 shows the numerics correctly reproducing the analytic result, with an instability with a maximum growthrate at around $k \approx 206$; this agrees well with the theoretical order-of-magnitude prediction of $k \approx\left(1-M^{2}\right)^{1 / 6} m^{-1 / 3} \delta^{-2 / 3} \approx 225$ from Ref. 33 . For large $k$ the numerics are overly dissipative, but this is as intended since such wavenumbers are poorly resolved spatially. Without the filtering, however, the characteristic method not only exhibits instability correctly at around $k \approx 206$, but also exhibits an artificial numerical instability of comparable growthrate at around $k \approx 625$, and another artificial numerical instability of much larger growthrate at around $k \approx 978$, very close to the Nyquist limit of $k=1047$. The reason for this behaviour is discussed in the next section.

\footnotetext{
${ }^{2}$ These DDA results have been verified against growth rates of the full time-domain simulations
} 


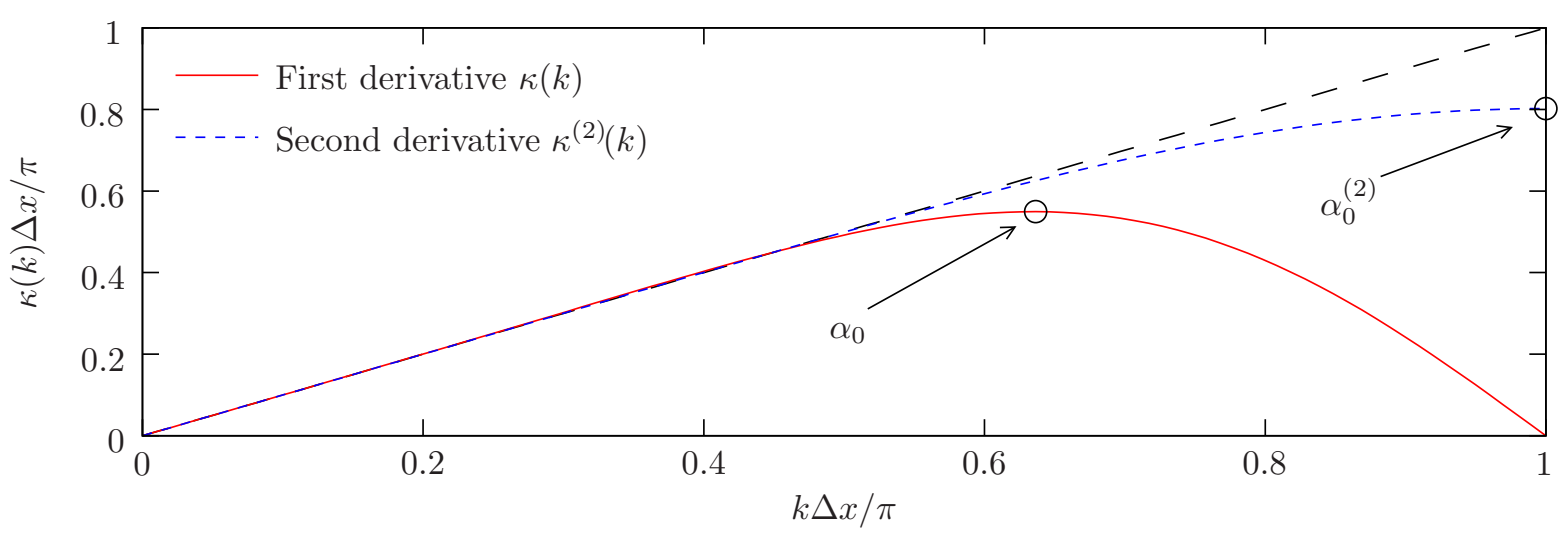

Figure 9: Effective wavenumbers $\kappa(k)$ and $\kappa^{(2)}(k)$ for the DRP finite difference derivatives used here. For reference $\kappa=k$ is also plotted. Circles indicate points where $\mathrm{d} \kappa / \mathrm{d} k=0$ and $\mathrm{d} \kappa^{(2)} / \mathrm{d} k=0$, given by $k \Delta x=\alpha_{0}$ and $k \Delta x=\alpha_{0}^{(2)}$ respectively.

\subsection{The cause of artificial instability}

To understand the stability of the discretized system shown in figure 8, we must first consider the limitations of the spatial differentiation used. For simplicity we consider only symmetric derivatives here, although the same argument will hold for nonsymmetric derivatives. The spatial derivatives used here are calculated using a finite-difference DRP scheme [11], such that if $f\left(x_{n}\right)=f_{n}$ for $x_{n}=n \Delta x$ then the derivative $f^{\prime}\left(x_{n}\right)$ is approximated by $f_{n}^{\prime}$, and the second derivative $f^{\prime \prime}\left(x_{n}\right)$ is approximated by $f_{n}^{\prime \prime}$, where

$$
f_{n}^{\prime}=\frac{1}{\Delta x} \sum_{j=1}^{N} a_{j}\left(f_{n+j}-f_{n-j}\right), \quad \quad f_{n}^{\prime \prime}=\frac{1}{(\Delta x)^{2}} \sum_{j=0}^{N^{(2)}} a_{j}^{(2)}\left(f_{n+j}+f_{n-j}\right)
$$

and $a_{j}$ and $a_{j}^{(2)}$ are constants that specify the scheme. Following the analysis of Tam and Webb [11], we consider a function $f(x)=\exp \{-\mathrm{i} k x\}$, and define the numerical wavenumbers $\kappa(k)=\mathrm{i} f_{n}^{\prime} / f_{n}$ and $\left(\kappa^{(2)}(k)\right)^{2}=-f_{n}^{\prime \prime} / f_{n}$, giving

$$
\kappa(k) \Delta x=2 \sum_{j=1}^{N} a_{j} \sin (k \Delta x) \quad\left(\kappa^{(2)}(k) \Delta x\right)^{2}=-2 \sum_{j=0}^{N^{(2)}} a_{j}^{(2)} \cos (k \Delta x) .
$$

(Note that in general $\kappa^{(2)} \neq \kappa$ apart from in the special case that the second derivative is formed by applying the first derivative stencil twice.) These numerical wavenumbers are plotted in figure 9] and are qualitatively representative of all finite-difference schemes; i.e. had different differentiation schemes been used, the functions $\kappa(k)$ and $\kappa^{(2)}(k)$ would still exhibit similar features. In particular, $\kappa=0$ at the Nyquist frequency $k \Delta x=\pi$, and hence $\mathrm{d} \kappa / \mathrm{d} k=0$ for some $k \Delta x=\alpha_{0}<\pi$. The sampling of the continuous solution introduces an aliasing of the numerical derivative; any wavenumber component such that $k \Delta x>\alpha_{0}$ is treated by the numerical derivative as the alias of a longer wavelength. This implies that any effective wavenumber $\kappa \Delta x$ is found for two different values of $k \Delta x$. The point $k \Delta x=\alpha_{0}$ corresponds to the transition from dissipative to parasitic damping referred to by Tam et al. [14]. For the DRP scheme used here [11], $\alpha_{0} \approx 0.634 \pi \approx 1.993$, or approximately 3 points per wavelength. Also, $\kappa^{(2)}$ exhibits a zero derivative when $k=\alpha_{0}^{(2)} / \Delta x$, which is at the Nyquist frequency $\alpha_{0}^{(2)}=\pi$ for symmetric stencils.

Since the continuous model being simulated is temporally unstable, there is at least one modal solution of the form $\exp \{\mathrm{i} \omega(k) t-\mathrm{i} k x\}$ for which $\operatorname{Im}(\omega(k))<0$. The numerical discretization would see this spatial wavenumber $k$ when calculating derivatives as if it were a spatial wavenumber $\kappa(k)$, and so we might expect the numerical scheme to support a comparable solution of the form $\exp \{\mathrm{i} \omega(\kappa(k)) t-\mathrm{i} k x\}$. This assumes the numerical time integration to be perfect (which is a reasonable assumption in our case, since the instabilities are well resolved in time, see [19]), and that $\kappa^{(2)}=\kappa$. If such a solution were supported, then owing to the zero derivative of $\kappa(k)$ at $k=\alpha_{0} / \Delta x$, the numerical solution would have 

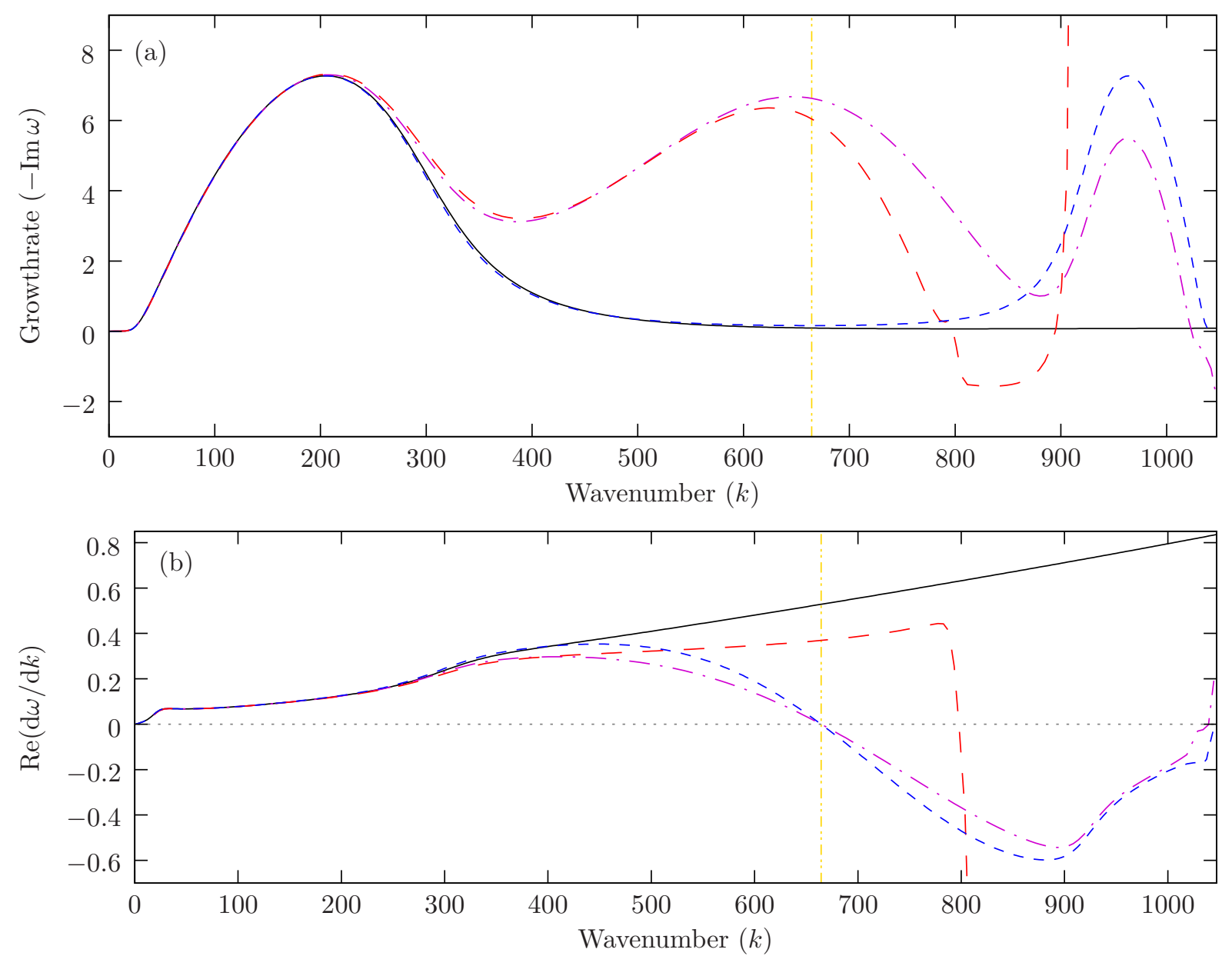

- Analytic $\omega(k) \quad-\cdots$ Analytic $\omega(\kappa(k)) \quad-\quad-$ Numerical $\quad-\cdot-$ Numerical $\left(D_{1}^{2}\right)$

Figure 10: Results of the Discrete Dispersion Analysis for grid 3 of case C. Two reference solutions $\omega(k)$ and $\omega(\kappa(k))$ from the analytic model are plotted against two numerical solutions using the characteristic boundary condition with no filtering. The $D_{1}^{2}$ numerical solution calculates the second derivative by applying the first derivative stencil twice. The vertical dot-dashed line denotes $k=\alpha_{0} / \Delta x$. The imaginary part of the group velocity, $\operatorname{Im}(\mathrm{d} \omega / \mathrm{d} k)$, is an order of magnitude smaller than the real part plotted in (b).

$\mathrm{d} \omega / \mathrm{d} k=0$ for $k=\alpha_{0} / \Delta x$; that is, the numerical solution would have an artificial zero group velocity at $k=\alpha_{0} / \Delta x$ with $\operatorname{Im}\left(\omega\left(\kappa\left(\alpha_{0} / \Delta x\right)\right)\right)<0$, potentially leading to an artificial absolute instability. This is what is seen in figure 4(b) for the Myers boundary condition.

In order to check these assumptions, figure 10 compares the growth rate and group velocity given by the theoretical dispersion relation $\omega(k)$, the semi-analytical expression $\omega(\kappa(k))$ and the DDA. For the latter the second derivative is calculated either by using directly the second-order derivative (13b) or by applying the first derivative stencil twice (labelled "Numerical $D_{1}^{2}$ " in figure 10], so that $\kappa^{(2)}=\kappa$.

The semi-analytic prediction $\omega(\kappa(k))$ describes accurately the theoretical instability at $k \simeq 200$. This is expected since in this range of wavenumbers the numerical dispersion error is small and $\kappa \simeq k$. More importantly, figure 10(a) shows the expression $\omega(\kappa(k))$ also captures the instability found in the numerical results at high wavenumbers. It is found at $k \simeq 910$, for which $\kappa(k) \simeq 200$, and is hence an alias of the theoretical instability at $k \simeq 200$. This indicates that the spurious instability $k \simeq 910$ in the numerical model is generated by the aliasing of the finite-difference stencil.

When the second derivative is calculated by applying the first-derivative stencil twice, the group velocity results from the DDA match the expression $\omega(\kappa(k))$ very well in figure 10(b). In particular, both show a zero group velocity at $k \Delta x \approx \alpha_{0}$ (unlike the group velocity of the theoretical dispersion relation which remains positive throughout). The growth rates in figure 10(a) also match closely, apart from around $k \Delta x \approx \alpha_{0}$ where the numerics support an additional instability. We therefore speculate here that this extra instability is due to the artificial zero group velocity at $k \Delta x=\alpha_{0}$, as theorized 


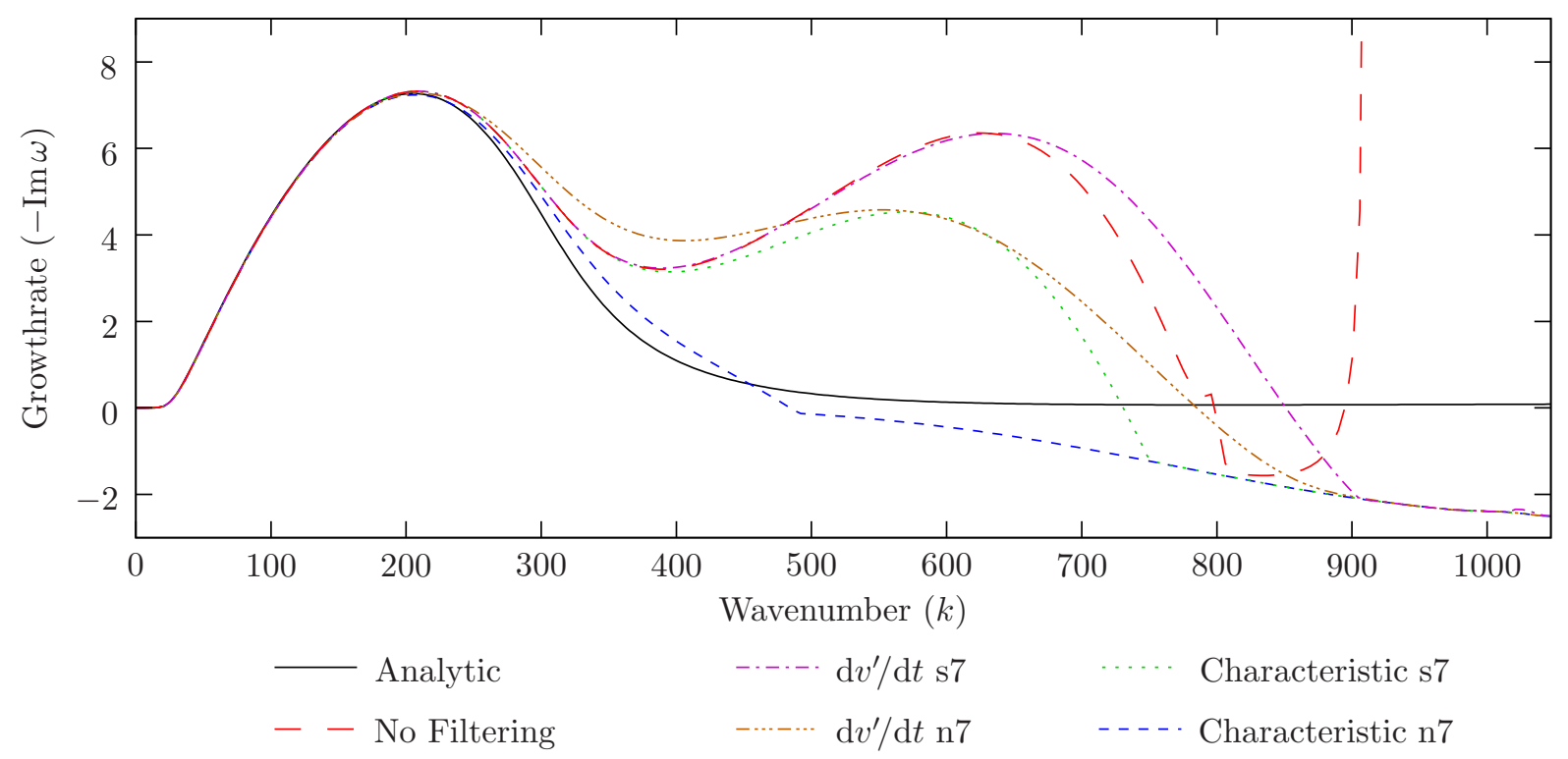

Figure 11: Growth rate against wavenumber for grid 3 of case C, applying the boundary condition using the characteristic method (11), for various different boundary condition filtering schemes. Either the normal velocity derivative $\mathrm{d} v^{\prime} / \mathrm{d} t$ or the characteristic amplitude $\theta$ is filtered using either the mild $\mathrm{s} 7$ or strong $\mathrm{n} 7$ filters described in $\lcm{5}$

above.

If instead the actual second derivative is used, rather than applying the first derivative twice, then as shown in figure 9 the second derivative has a zero derivative of $\kappa^{(2)}$ at $k \Delta x=\alpha^{(2)}=\pi$, and this gives rise to the far more extreme instability near the Nyquist frequency seen in figure 10(a) (for the curve labelled "Numerical").

In summary, this section motivates the use of spatial filtering within the boundary condition that is very strong around the wavenumbers $\alpha_{0}$ and $\alpha_{0}^{(2)}$.

\subsection{Methods of filtering the boundary condition}

The previous section suggests numerical filtering of the boundary condition is important to remove artificial numerical instabilities while retaining real instabilities of the underlying continuous model. Here, we consider the effect of several different ways of filtering the boundary condition. While 55 discusses the filters used, here for demonstration purposes we will make use of a standard 7-point 6th order low pass filter (s7) and a more dissipative 7-point 4th order low pass filter (n7). These filters could be applied to either the calculated boundary velocity derivative $\mathrm{d} v^{\prime} / \mathrm{d} t$ (as in equation 12) or to the amplitude of the incoming characteristic $\theta$ (as in equation 111). Each of these possibilities is plotted in figure 11, and the only combination that correctly reflects the actual stability of the continuous system is filtering the characteristic amplitude $\theta$ using the $\mathrm{n} 7$ filter. If $\mathrm{d} v^{\prime} / \mathrm{d} t$ were filtered, then at high wavenumbers the boundary condition would effectively be that of a hard perfectly reflecting boundary with $\mathrm{d} v^{\prime} / \mathrm{d} t=0$. If instead the incoming characteristic $\theta$ is filtered, then at high wavenumbers the boundary condition would effectively be that of no incoming characteristic with $\theta=0$; i.e. an absorbing boundary. However, even when filtering the incoming characteristic, the s7 filter is not strong enough around $k=\alpha_{0} / \Delta x$ to completely suppress the artificial instability due to $\mathrm{d} \kappa / \mathrm{d} k=0$, and so some instability is still seen around $k \approx 600$ in figure 11]. Only the n7 filter is sufficiently strong around $k \Delta x=\alpha_{0}$ to give the correct stability behaviour of the underlying continuous system, and even then only when applied to the incoming characteristic amplitude $\theta$. Because of this, the numerical results shown in $₫ 3$ where obtained using the $\mathrm{n} 7$ filter applied to the incoming characteristic amplitude. 


\begin{tabular}{r|cccccc} 
filter & width $(2 N+1)$ & order at $0\left(n_{0}\right)$ & order at $\pi\left(n_{\pi}\right)$ & pinned to 1 at & weighting $(R)$ & $\hat{\mathcal{F}}\left(\alpha_{0}\right)$ \\
\hline s7 & 7 & 6 & 2 & $\times$ & - & 0.35 \\
n7 & 7 & 4 & 4 & $\times$ & - & 0.79 \\
p11 & 11 & 6 & 2 & $2 \pi / 3$ & - & 0.99 \\
w15 & 15 & 6 & 2 & $3 \pi / 4$ & 1 & 0.82 \\
p17 & 17 & 6 & 4 & $2 \pi / 3$ & $1 / 2$ & 0.98
\end{tabular}

Table 2: Optimization parameters used for finding optimized filters. A cross denotes a value not used, and a dash denotes a value having no effect on the filter coefficients. $\hat{\mathcal{F}}\left(\alpha_{0}\right)$ shows the effect of the filter at the zero group velocity wavenumber $\alpha_{0} \approx 1.993$ for the DRP scheme used here. The coefficients obtained are given in table 3

\section{Design and performance of numerical filtering at the bound- ary}

The filter for the boundary condition should be designed to avoid the artificial absolute instability due to the zero derivative of $\kappa(k)$ at $k \Delta x=\alpha_{0}$, and to suppress the copy of the theoretical instability due to aliasing for $k \Delta x>\alpha_{0}$. This low-pass filter $(1-\mathcal{F})$ is introduced into the boundary condition by filtering the incoming characteristic amplitude (11). It is significantly less computationally expensive than the filtering applied throughout the fluid, since it is only applied at the comparatively few locations lying along an impedance boundary. Since this filter is applied to the incoming characteristic which is calculated afresh at each time step, the filter is applied with strength 1 , in contrast to the filtering throughout the fluid which is typically applied with a small amplitude owing to the cumulative effect of repeatedly filtering at each timestep. The filter $\mathcal{F}$ is implemented numerically as a $(2 N+1)$-point symmetric finite difference filter, so that a quantity $f_{n}=f(n \Delta x)$ is filtered to $f_{n}^{f}$, where

$$
f_{n}^{f}=f_{n}-\underbrace{\sum_{j=-N}^{N} d_{|j|} f_{n+j}}_{\mathcal{F} f} .
$$

Following Tam et al. [14], assuming $f(x)=\hat{f} \exp \{-\mathrm{i} k x\}$ gives

$$
\hat{f}^{f}=\hat{f}-\hat{\mathcal{F}}(k \Delta x) \hat{f} \quad \text { where } \quad \hat{\mathcal{F}}(\alpha)=d_{0}+\sum_{j=1}^{N} 2 d_{j} \cos (j \alpha) .
$$

We now consider possible coefficients $d_{j}$ to optimize $\hat{\mathcal{F}}$. We require $\hat{\mathcal{F}}(0)=0$ and $\hat{\mathcal{F}}(\pi)=1$, and in addition $\mathrm{d}^{j} \hat{\mathcal{F}} / \mathrm{d}^{j}=0$ at $\alpha=0$ for $j=1, \ldots, n_{0}-1$ and at $\alpha=\pi$ for $j=1, \ldots, n_{\pi}-1$ to give an $n_{0}$ order filter with order $n_{\pi}$ damping at the Nyquist frequency $\alpha=\pi$. Inspired by the test filters of Bogey and Bailly [12], here we require the filter to be optimal in the sense that it minimizes

$$
M\left(d_{j}\right)=R \int_{\pi / 16}^{\pi / 3}|\hat{\mathcal{F}}(\alpha)| \frac{\mathrm{d} \alpha}{\alpha}+\int_{2 \pi / 3}^{\pi}|1-\hat{\mathcal{F}}(\alpha)| \frac{\mathrm{d} \alpha}{\alpha},
$$

where $R$ is a weighting parameter to prefer low damping at small wavenumbers against high damping at large wavenumbers. We require $0 \leq \hat{\mathcal{F}}(\alpha)$, and unlike the test filters of Ref. 12, we also require $\hat{\mathcal{F}}(\alpha) \leq 1$, which was found necessary to ensure stability in our application. We also have the additional restriction that the filter should be sufficiently damping at the zero derivative wavenumber $\alpha_{0}$ that the artificial instability is suppressed. Since in general the amount of damping necessary at $\alpha_{0}$ will depend on the stability of the boundary at the wavelength $\alpha_{0} / \Delta x$, and will therefore vary with $\Delta x$, one possibility to ensure sufficient damping for arbitrary $\Delta x$ is to pin the filter to satisfy $\hat{\mathcal{F}}\left(\alpha_{0}\right)=1$. However, $\alpha_{0}$ varies with the derivative scheme used, so that, for example, the higher order derivative schemes of Bogey and Bailly [12] have different values for $\alpha_{0}$. Another possibility, pursued here, is therefore to pin $\hat{\mathcal{F}}=1$ at some suitable value of $\alpha$, and we find $\hat{\mathcal{F}}(2 \pi / 3)=1$ and $\hat{\mathcal{F}}(3 \pi / 4)=1$ lead to efficient filters.

Several filtering schemes are proposed here, with their parameters listed in table 2 and their coefficients $d_{j}$ given in table 3 The s7 filter is a standard 7-point 6th order filter for comparison purposes, while the $\mathrm{n} 7$ filter is a similar 7-point filter but relaxed to 4 th order to allow greater damping at short 
(a)

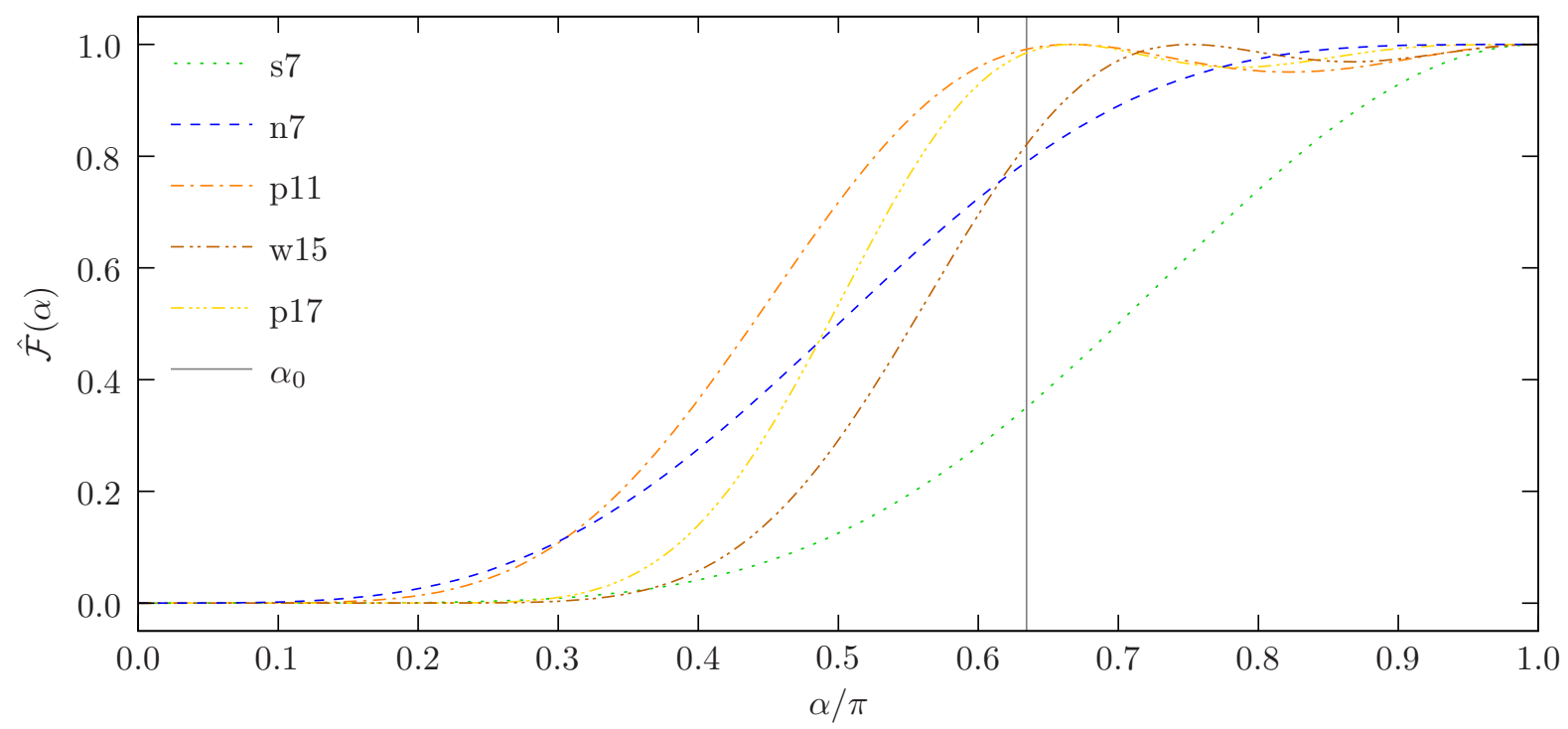

(b)

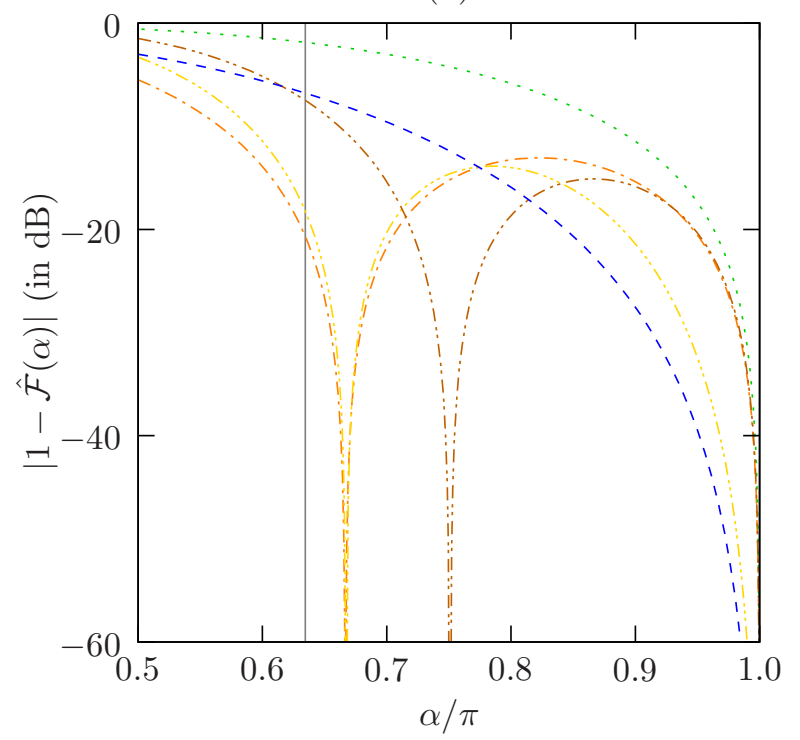

(c)

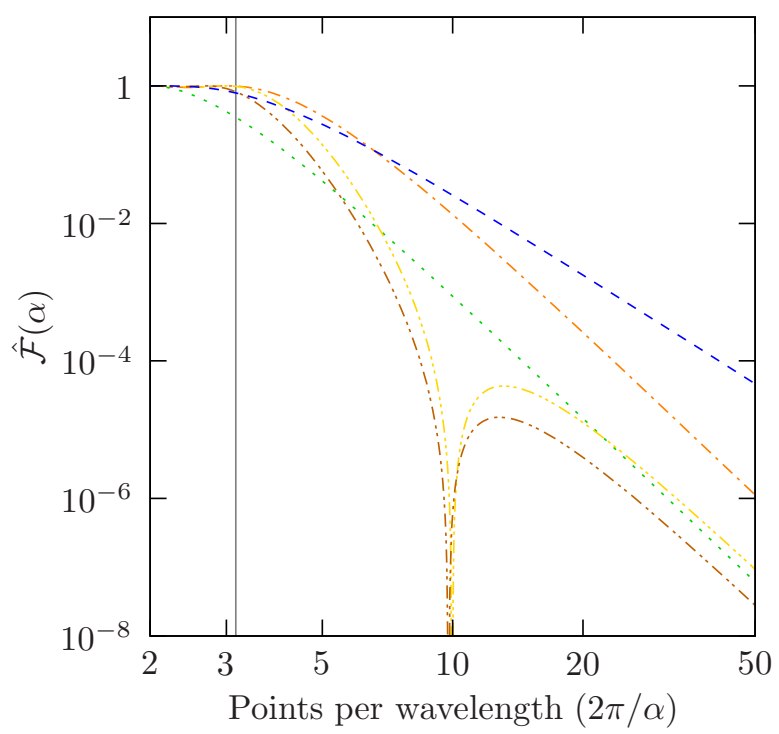

Figure 12: Plots of the response of the filters listed in table 2 defined by (15) with coefficients given in table 3 The vertical line labelled $\alpha_{0}$ denotes the position of the zero derivative of the DRP derivative stencil. (a) The overall response of the filter. (b) Overall damping (in $\mathrm{dB}$ ) at short wavelengths. (c) Effect of damping against number of points per wavelength.

wavelengths. The p11 and p17 filters are 11- and 17-point 6th order filters respectively, pinned to give complete filtering at $\alpha=2 \pi / 3$, and therefore giving almost complete filtering at $\alpha=\alpha_{0}$ for the DRP derivatives used here (as seen from the last column in table 22). The w15 filter is similarly a 15-point 6 th order filter, but pinned to give complete filtering at $\alpha=3 \pi / 4$. The s7, $\mathrm{n} 7$ and p11 filters are completely specified by their constraints, and therefore only the w15 and p17 filters have spare degrees of freedom that can be used to minimize $M\left(d_{j}\right)$. Plots of these filters are given in figure 12, If the stencil width of the filter is of no concern, the p17 filter gives both a high level of filtering at $\alpha_{0}$ and shorter wavelengths, and a very low level of dissipation for longer wavelengths, having an effect of magnitude less than $10^{-3}$ for more than 8 points per wavelength and an effect of less than $10^{-4}$ for more than 9 points per wavelength (see figure 12(c)). For the 7-point filters and the w15 filter, care must be taken to ensure the filtering at $\alpha_{0}$ is sufficient to prevent the artificial absolute instability.

Figure 13 plots the performance of these filters applied to the incoming characteristic for case C grid 4, using the discrete dispersion analysis (DDA). All filters successfully remove the large artificial instability near the Nyquist frequency $k \Delta x=\pi$, since all filters are required to give total dissipation 


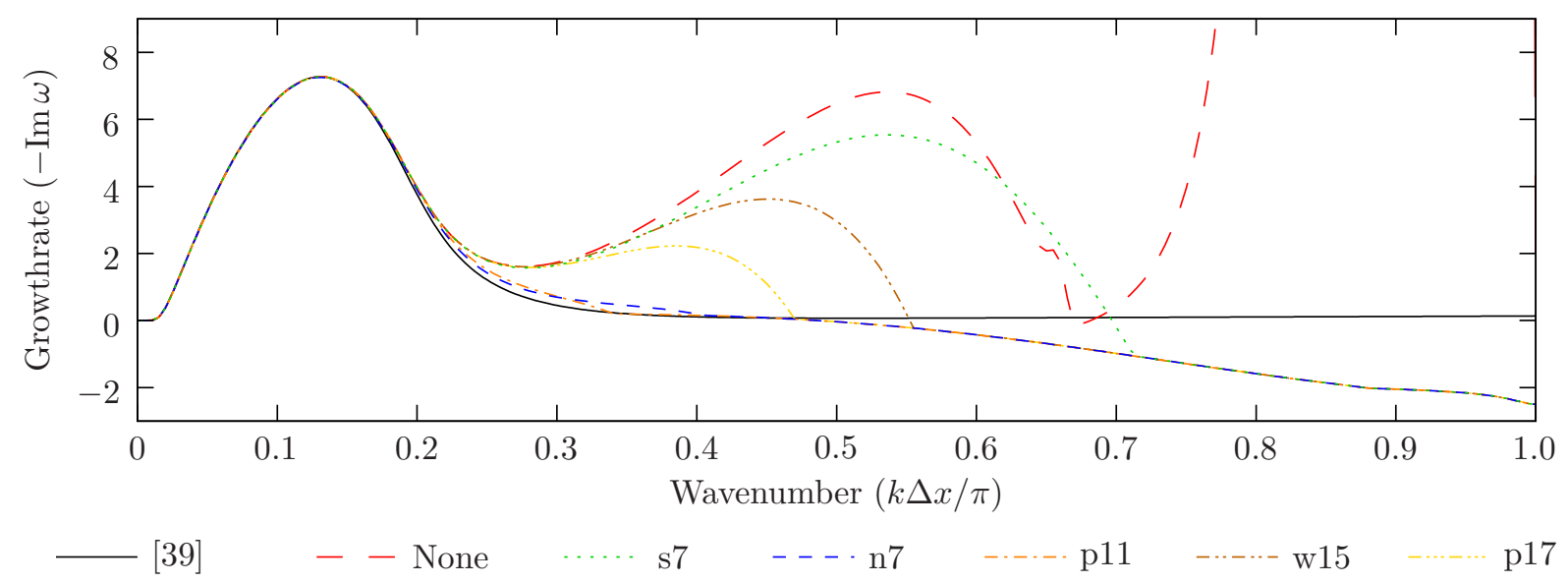

Figure 13: For case C, grid 4, plots of the growth rate $(-\operatorname{Im} \omega)$ predicted by the DDA 19] against wavenumber $k$ for various filters, together with the analytic solution using the modified boundary condition from Ref. 39 .

there. Also, all filters correctly reproduce the real instability at low wavenumbers. However, the only filters to completely remove the artificial instability for mid wavenumbers are the $\mathrm{n} 7 \mathrm{and}$ p11 filters, since these are the most dissipative filters at wavenumbers of the order $k \Delta x \approx \pi / 4$. Since the p11 filter uses a significantly wider stencil than the $\mathrm{n} 7$ filter, the $\mathrm{n} 7$ filter has been used for all the numerical results in 93 The p11 filter may however be useful when the continuous system is even more unstable because the p11 filter, being pinned to be totally dissipative at $k \Delta x=2 \pi / 3$, is a significantly stronger filter around the artificial instability wavenumber $\alpha_{0}$ than the $\mathrm{n} 7$ filter, as shown by the last column in table 2

The more advanced w15 and p17 filters are seen not to filter sufficiently strongly in the mid range of wavenumbers. While further investigation and careful choice of optimization function may yield a better performing wide filter, we believe the analysis in this section suggests there is little to be gained from such an investigation, and that a strong simple filter such as the $\mathrm{n} 7$ filter (or, if need be, the p11 filter) should be sufficient for many typical applications.

\section{Conclusion}

This paper presents a time-domain version of the frequency-domain modified boundary condition of Ref. 25, given by equation (9). A reference implementation is also given by directly calculating (9) and applying it using (11), using the filter $\mathcal{F}$ given by (15) with coefficients $\mathrm{n} 7$ from table 3 . The use of characteristic boundary conditions (11) was found particularly important. While the reference implementation is only demonstrated for a simple two-dimensional homentropic uniform flow over a flat impedance boundary, the boundary condition derived and its method of application are immediately applicable to three-dimensional non-homentropic nonuniform flows. The are no technical barriers to using the technique with a curved impedance boundary, although since the underlying boundary condition of Ref. 25 was derived under the assumption of a flat impedance boundary and lacks an equivalent of the $\boldsymbol{n} \cdot \nabla \boldsymbol{u}_{\mathbf{0}} \cdot \boldsymbol{n}$ curvature term in the Myers boundary condition (11), the accuracy of computations using the proposed boundary condition on a curved impedance boundary are not guaranteed.

The goal of the modified boundary condition of Ref. 25 was to find a mathematically well-posed boundary condition that allows for a stability analysis, and which it was hoped would allow convergence of numerical simulations with grid refinement; a particular deficiency of the Myers boundary condition 20]. The reference implementation given here shows that indeed convergence with grid refinement is possible (figure 17), and that, if care is taken with how the boundary condition is implemented, the stability properties of the underlying continuous model can be reproduced numerically. The reference implementation also demonstrates how to deal with certain artifacts of the numerical discretization which are believed to be applicable to numerical simulations of unstable systems in general. These artifacts are caused by the aliasing of the theoretical instability, and by the artificially induced zero group velocity of the spatial discretization at the wavenumber $k=\alpha_{0} / \Delta x$, which combines with the temporally unstable continuous system being discretized to produce an artificial absolute instability. Both of these effects 
depend on the spatial finite difference scheme used. Strong but precise filtering of the boundary condition is needed to suppress these artificial instabilities while retaining any physical instabilities. Such filtering will also depend on the spatial scheme used, although we believe the approximation $\alpha_{0} \approx 2 \pi / 3$ assumed for several of the filters derived in section 5 will commonly be valid. The artificial zero group velocity causes no problems if the wavenumber $k=\alpha_{0} / \Delta x$ is stable, and therefore this feature is not seen for numerical simulations of stable systems.

It should however be noted that the underlying continuous model [25] is only an approximation of the Pridmore-Brown equation (as for example plotted in figure 6) valid for small boundary layer thicknesses $\delta$ and not-too-high wavenumbers $k \ll 1 / \delta$, and even the Pridmore-Brown equation is only an inviscid approximation to the true boundary layer behaviour. This means that perfectly reproducing the continuous approximation of Ref. 25 using a very fine discretization is not necessarily desirable. The restriction to $k \ll 1 / \delta$ is not limiting here, since to resolve such high wavenumbers would require a discretization $\Delta x=O(\delta)$ which would therefore be sufficiently fine to resolve the boundary layer explicitly without having to resort to a modified boundary condition. Indeed, Ref. 33 predicts that the most unstable behaviour occurs at a wavenumber with an order of magnitude $k \approx\left(1-M^{2}\right)^{1 / 6} m^{-1 / 3} \delta^{-2 / 3}$, which agrees well with the results given here, and therefore suggests that $\Delta x \approx\left(1-M^{2}\right)^{-1 / 6} m^{1 / 3} \delta^{2 / 3}$ should be sufficient to correctly resolve the stability behaviour of the underlying continuous model.

All the results presented here have reproduced the convectively unstable behaviour of the underlying inviscid continuous model; indeed, it was proposed in Ref. 25 that inviscid flows over non-rigid boundaries "are absolutely unstable for sufficiently thin boundary layers, and are convectively unstable otherwise". In contrast, most modern computational aeroacoustics simulations assume stability, either explicitly or implicitly, and then tune the artificial numerical filtering they apply in order to achieve this, irrespective of whether the equations being solved support an instability. One has only to think of the flapping of a flag in the wind [32] to realize that flow over non-rigid surfaces may be physically unstable, and there is growing experimental evidence that flows over acoustic linings also exhibit an instability [30, 31]. Incorporating viscosity into the boundary layer over acoustic linings affects the stability of the flow, and can completely stabilize the flow provided viscosity is strong enough [36]. A boundary condition incorporating this stabilizing effect of viscosity is currently being investigated [42], and it is expected that the analysis provided here may be applied to this and other modified boundary conditions in future to yield the correct stability behaviour of the underlying model. Even in the inviscid case, time-domain simulations that include convective instability are not problematic provided the acoustic liner has a finite length (unlike the infinite-length liner considered here), meaning that the instability can only grow to a certain magnitude before reaching the end of the liner. The feedback mechanism generated by an instability over a finite-length liner scattering into acoustic waves at the liner trailing edge and triggering the instability once they reach the liner leading edge may therefore be analysed in time-domain simulations using the method proposed in this paper.

A number of optimized filters were considered here in $\$ 5$, with the simple seven-point fourth-order filter $\mathrm{n} 7$ performed best in practice while also having the advantage of a small stencil. Due to this filtering, about ten points per wavelength were necessary for numerical resolution at the boundary, and so the better performance of highly optimized DRP stencils at fewer points per wavelength was wasted at the boundary. One possibility for future research would be to reoptimize the coefficients of a finitedifference stencil in a similar way as for a DRP scheme, but optimizing to achieve the largest possible value of $\alpha_{0}$. This would allow for filters to be reoptimized for this new value of $\alpha_{0}$ that filter more weakly at smaller wavenumbers, and thus could possibly allow for accurate simulations with fewer points per wavelength. However, we emphasise that the optimization of the derivative stencil and the filter are linked such that both need optimizing together to give a coherent optimal numerical scheme.

There are a number of other possible extensions to the work presented here. The boundary layer assumed here has a uniform density and a linear velocity variation, while the modified boundary condition of Ref. 25 allows for arbitrary boundary layer profiles of velocity and density. In addition, the analysis here assumes a flat impedance surface, where as the Myers boundary condition [8] allows for arbitrary curvature. The boundary layer was also considered to be inviscid, and other boundary conditions that include viscosity 34, 35, 38, 42, 43] could also be investigated. Numerically, we assume a finite difference numerical scheme with a constant grid spacing $\Delta x$, and either a variable grid spacing or indeed a different scheme such as a finite element simulation could be considered. 


\section{Acknowledgements}

E.J. Brambley gratefully acknowledges the support of the Royal Society through a University Research Fellowship, and Fellowship of Gonville \& Caius College, Cambridge. A preliminary version of some parts of this paper was presented as part of AIAA Paper 2013-2218 at the 19th AIAA/CEAS Aeroacoustics Conference in Berlin, Germany [1].

\section{Appendix A. Conservative form of the Euler and linearized Eu- ler equations}

We consider fluid with velocity $\boldsymbol{u}$, pressure $p$, density $\rho$, temperature $T$ and specific entropy $s$. Neglecting viscosity and heat transfer, the governing equations are the Euler equations, written in their conservative form as

$$
\begin{aligned}
\frac{\partial \rho}{\partial t}+\nabla \cdot(\rho \boldsymbol{u}) & =Q \\
\frac{\partial}{\partial t}(\rho \boldsymbol{u})+\nabla \cdot(\rho \boldsymbol{u} \boldsymbol{u}+p \mathbf{l}) & =\boldsymbol{f}+Q \boldsymbol{u} \\
\frac{\partial}{\partial t}(\rho s)+\nabla \cdot(\rho s \boldsymbol{u}) & =S+Q s
\end{aligned}
$$

where I is the identity matrix, and $Q, S$, and $f$ are a mass source, entropy source, and body force respectively. Note that the entropy $s$ is only defined up to addition of an arbitrary constant in this formulation. We now suppose the flow consists of small perturbations to a steady mean flow $\boldsymbol{u}_{0}, p_{0}$, $\rho_{0}, s_{0}$, and we denote small perturbations by a prime, so that $\rho^{\prime}=\rho-\rho_{0}$ is the small time-dependent density perturbation and $(\rho \boldsymbol{u})^{\prime}=\rho \boldsymbol{u}-\rho_{0} \boldsymbol{u}_{0}$ is the small time-dependent momentum perturbation. The small perturbations are governed by the Linearized Euler Equations (LEE), which may be written as

$$
\frac{\partial \boldsymbol{q}}{\partial t}+\sum_{i} \frac{\partial}{\partial x_{i}}\left(\mathrm{~F}_{i} \boldsymbol{q}\right)=\boldsymbol{H}
$$

where, in cartesian coordinates $\boldsymbol{u}=u \boldsymbol{e}_{x}+v \boldsymbol{e}_{y}+w \boldsymbol{e}_{z}$,

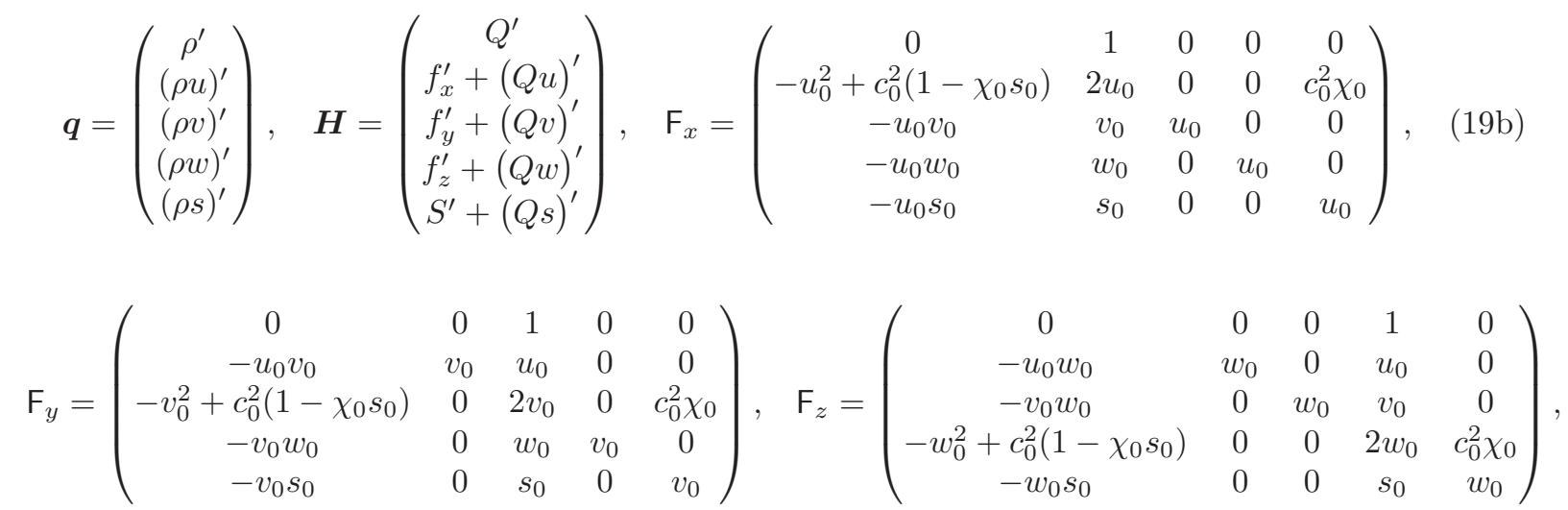

where $c^{2}=\left.\frac{\partial p}{\partial \rho}\right|_{s}$ is the square of the speed of sound, $\beta=-\left.\frac{1}{\rho} \frac{\partial \rho}{\partial T}\right|_{p}$ is the coefficient of thermal expansion, $\mathrm{c}_{p}$ is the specific heat at constant pressure, and $\chi=\beta T / \mathrm{c}_{p}$. For a perfect gas with ratio of specific heats $\gamma=\mathrm{c}_{p} / \mathrm{c}_{v}$, these are given by $c^{2}=\gamma p / \rho$ and $\beta=1 / T$. The vectors $\mathrm{F}_{i} \boldsymbol{q}$ may be interpreted as the flux of $\boldsymbol{q}$ in the $\boldsymbol{e}_{\boldsymbol{i}}$ direction, and hence $\mathrm{F}(\boldsymbol{n}) \boldsymbol{q}=\sum_{i} n_{i} \mathrm{~F}_{i} \boldsymbol{q}$ gives the flux of $\boldsymbol{q}$ is the $\boldsymbol{n}$ direction. The eigenvectors of $\mathrm{F}(\boldsymbol{n})=\sum_{i} n_{i} \mathrm{~F}_{i}$ give the characteristics of the LEE in the direction $\boldsymbol{n}$, and the corresponding eigenvalues give the speed of propagation along those characteristics. For example, the 
characteristics in the $\boldsymbol{e}_{y}$ direction are given by the columns of $\mathrm{W}_{y}$, where

$$
\begin{aligned}
& \mathrm{F}_{y}=\mathrm{W}_{y} \mathrm{D}_{y} \mathbf{W}_{y}^{-1}, \mathrm{D}_{y}=\operatorname{diag}\left(v_{0}, v_{0}, v_{0}, v_{0}+c_{0}, v_{0}-c_{0}\right), \\
& \mathbf{W}_{y}=\left(\begin{array}{ccccc}
1 & 0 & 0 & 1 & 1 \\
0 & 1 & 0 & u_{0} & u_{0} \\
v_{0} & 0 & 0 & v_{0}+c_{0} & v_{0}-c_{0} \\
0 & 0 & 1 & w_{0} & w_{0} \\
s_{0}-\frac{1}{\chi_{0}} & 0 & 0 & s_{0} & s_{0}
\end{array}\right), \quad \mathbf{W}_{y}^{-1}=\left(\begin{array}{ccccc}
\chi_{0} s_{0} & 0 & 0 & 0 & -\chi_{0} \\
u_{0}\left(\chi_{0} s_{0}-1\right) & 1 & 0 & 0 & -u_{0} \chi_{0} \\
w_{0}\left(\chi_{0} s_{0}-1\right) & 0 & 0 & 1 & -w_{0} \chi_{0} \\
\frac{c_{0}-v_{0}}{2 c_{0}}-\frac{\chi_{0} s_{0}}{2} & 0 & \frac{1}{2 c_{0}} & 0 & \frac{\chi_{0}}{2} \\
\frac{c_{0}+v_{0}}{2 c_{0}}-\frac{\chi_{0} s_{0}}{2} & 0 & \frac{-1}{2 c_{0}} & 0 & \frac{\chi_{0}}{2}
\end{array}\right) .
\end{aligned}
$$

The first column of $\mathrm{W}_{y}$ defines the characteristic corresponding to entropy perturbations and the second and third columns correspond to vortical perturbations, all convected with the fluid at velocity $v_{0}$, while the final two columns of $\mathrm{W}_{y}$ correspond to acoustic perturbations propagating at velocity $c_{0}$ relative to the mean flow velocity $v_{0}$. The amplitudes of these characteristics are given by $\mathbf{W}_{y}^{-1} \boldsymbol{q}$ which corresponds to the projection of the solution $\boldsymbol{q}$ on the vector basis $\mathbf{W}_{y}$. For a perfect gas $\left(\beta_{0}=1 / T_{0}\right)$ with a homentropic base flow $\left(s_{0}\right.$ constant) we may choose the arbitrary constant of $s_{0}$ at will; choosing $s_{0}=\mathrm{c}_{p}$ significantly simplifies (19) and (20) in this case.

\section{Appendix A.1. Characteristic boundary condition}

The boundary conditions (5) or (9) specify the normal velocity $v^{\prime}$ and are used here to calculate the incoming characteristics. To that end the time derivative $\partial \boldsymbol{q}^{\mathrm{bc}} / \partial t$ used to march the solution on the boundary forward in time is written

$$
\frac{\partial \boldsymbol{q}^{\mathrm{bc}}}{\partial t}=\mathrm{W}_{y} \mathrm{PW}_{y}^{-1} \frac{\partial \boldsymbol{q}}{\partial t}+\theta_{y}^{-} \boldsymbol{w}_{y}^{-}, \text {where } \quad \mathrm{P}=\operatorname{diag}(1,1,1,1,0)
$$

where $\partial \boldsymbol{q} / \partial t$ is the time derivative calculated with the LEE (19a). The projection operator $\mathrm{W}_{y} \mathrm{PW}_{y}^{-1}$ eliminates the incoming characteristic. The final column of $\mathbf{W}_{y}$ is denoted by $\boldsymbol{w}_{y}^{-}$and $\theta_{y}^{-}$is the rate of change of the incoming characteristic that will be obtained from the boundary condition.

Using equation (20) for $\mathrm{W}_{y}$ (simplified with the mean flow boundary condition $v_{0}=0$ ) we can write the incoming characteristic in terms of the imposed normal velocity $v^{\prime}$ and the outgoing characteristic $\theta_{y}^{+}$:

$$
\theta_{y}^{-}=\theta_{y}^{+}-\frac{\rho_{0}}{c_{0}} \frac{\partial v^{\prime}}{\partial t}
$$

The rate of change of the outgoing characteristic is given by $\theta_{y}^{+}=\boldsymbol{p}_{y}^{+} \cdot \partial \boldsymbol{q} / \partial t$ where $\boldsymbol{p}_{y}^{+}$is the fourth row of $\mathrm{W}_{y}^{-1}$. Combining (21) and (22) we can write

$$
\frac{\partial \boldsymbol{q}^{\mathrm{bc}}}{\partial t}=\frac{\partial \boldsymbol{q}}{\partial t}-\left[\boldsymbol{p}_{y}^{-} \cdot \frac{\partial \boldsymbol{q}}{\partial t}-(1-\mathcal{F}) \theta_{y}^{-}\right] \boldsymbol{w}_{y}^{-} \quad \text { with } \quad \theta_{y}^{-}=\boldsymbol{p}_{y}^{+} \cdot \frac{\partial \boldsymbol{q}}{\partial t}-\frac{\rho_{0}}{c_{0}} \frac{\partial v^{\prime}}{\partial t}
$$

where $\boldsymbol{p}_{y}^{-}$is the fifth row of $\mathbf{W}_{y}^{-1}$ and we have introduced the optional spatial filtering $\mathcal{F}$.

Assuming a perfect gas $\left(\beta_{0}=1 / T_{0}\right)$ with a homentropic base flow $\left(s_{0}=c_{p}\right)$, rewriting (23) in terms of primitive variables by using, e.g., $(\rho u)^{\prime}=\rho_{0} u^{\prime}+\rho^{\prime} u_{0}$ leads to the characteristic boundary condition as given in (11).

\section{Appendix B. Details of the two-dimensional test case}

Consider the two-dimensional problem, depicted in figure 1] of a perfect gas occupying $y<0$ with constant density $\rho_{0} \equiv 1$, constant sound speed $c_{0} \equiv 1$, constant entropy $s_{0}=\mathrm{c}_{p}$, constant pressure $p_{0}$ and constant velocity $\boldsymbol{u}_{0}=M \boldsymbol{e}_{x}$. A small oscillatory point mass source of strength $Q^{\prime}=\delta(x) \delta\left(y+y_{s}\right) \sin (\omega t)$ generates small perturbations to this steady flow, and the wall at $y=0$ responds as a mass-springdamper boundary governed by (2). The governing equations (19), together with a Perfectly Matched Layer (PML) absorbing boundary [40] along the bottom boundary at $y=-H$, and the Myers (5) or modified (9) boundary conditions enforced in terms of characteristics (22) along the top boundary $y=0$, lead to

$$
\frac{\partial \boldsymbol{q}}{\partial t}=-\mathrm{F}_{x} \frac{\partial \boldsymbol{q}}{\partial x}-\mathrm{F}_{y} \frac{\partial \boldsymbol{q}}{\partial y}-\sigma_{y} \boldsymbol{q}-\sigma_{y} \mathrm{~F}_{x} \frac{\partial \check{\boldsymbol{q}}}{\partial x}+\boldsymbol{H},
$$




\begin{tabular}{l|rcccc} 
& \multicolumn{1}{|c}{$\mathrm{s} 7$} & $\mathrm{n} 7$ & $\mathrm{p} 11$ & $\mathrm{w} 15$ & $\mathrm{p} 17$ \\
\hline$d_{0}$ & $5 / 16$ & $1 / 2$ & $241 / 432$ & +0.44791875517975 & +0.50550728999206 \\
$d_{1}$ & $-15 / 64$ & $-9 / 32$ & $-499 / 1728$ & -0.29901645275196 & -0.30412324388831 \\
$d_{2}$ & $3 / 32$ & 0 & $-47 / 864$ & +0.03909040448289 & -0.01070299832430 \\
$d_{3}$ & $-1 / 64$ & $1 / 32$ & $47 / 864$ & +0.06686926971278 & +0.07842585728816 \\
$d_{4}$ & & & $11 / 432$ & -0.02610492651421 & +0.00845081979867 \\
$d_{5}$ & & & $-1 / 64$ & -0.01513804428420 & -0.03287994103697 \\
$d_{6}$ & & & & +0.01305514444145 & +0.00215383104915 \\
$d_{7}$ & & & & -0.00271477267662 & +0.00857732763713 \\
$d_{8}$ & & & & & -0.00265529751954
\end{tabular}

Table 3: Coefficients for the filters given in table 2

where

$\boldsymbol{q}=\left(\begin{array}{c}\rho^{\prime} \\ (\rho u)^{\prime} \\ (\rho v)^{\prime} \\ p^{\prime}\end{array}\right), \quad \boldsymbol{H}=\delta(x) \delta\left(y+y_{s}\right) \sin (\omega t)\left(\begin{array}{c}1 \\ M \\ 0 \\ 1\end{array}\right), \quad \mathrm{F}_{x}=\left(\begin{array}{cccc}0 & 1 & 0 & 0 \\ -M^{2} & 2 M & 0 & 1 \\ 0 & 0 & M & 0 \\ -M & 1 & 0 & M\end{array}\right), \quad \mathrm{F}_{y}=\left(\begin{array}{cccc}0 & 0 & 1 & 0 \\ 0 & 0 & M & 0 \\ 0 & 0 & 0 & 1 \\ 0 & 0 & 1 & 0\end{array}\right)$,

$$
\frac{\partial \check{\boldsymbol{q}}}{\partial t}=\boldsymbol{q}, \quad \sigma_{y}=\left\{\begin{array}{ll}
\frac{2}{\Delta y}\left|1-\frac{H+y}{H_{\mathrm{PML}}}\right|^{2} & -H \leq y \leq-H+H_{\mathrm{PML}} \\
0 & y>-H+H_{\mathrm{PML}}
\end{array} \quad, \quad H_{\mathrm{PML}}=\left(N_{\mathrm{PML}}-1\right) \Delta y\right.
$$

Periodic boundary conditions are used at the upstream and downstream ends of the domain $x=L^{ \pm}$. On the impedance boundary $y=0$ the time derivative of the solution is calculated as follows:

$$
\frac{\partial \boldsymbol{q}^{\mathrm{bc}}}{\partial t}=\frac{\partial \boldsymbol{q}}{\partial t}-\left[\boldsymbol{p}_{y}^{-} \cdot \frac{\partial \boldsymbol{q}}{\partial t}-(1-\mathcal{F}) \theta_{y}^{-}\right]\left(\begin{array}{c}
1 \\
M \\
-1 \\
1
\end{array}\right)
$$

with

$$
\begin{gathered}
\theta_{y}^{-}=\boldsymbol{p}_{y}^{+} \cdot \frac{\partial \boldsymbol{q}}{\partial t}-\frac{\partial v^{\prime}}{\partial t}, \\
\boldsymbol{p}_{y}^{ \pm}=\frac{1}{2}\left(\begin{array}{cccc}
0 & 0 & \pm 1 & 1
\end{array}\right), \\
\frac{\partial v^{\prime}}{\partial t}=\frac{\partial v_{s}}{\partial t}+M \frac{\partial v_{s}}{\partial x}+\delta M\left[\frac{\partial^{2}}{\partial x^{2}}\left((\rho u)^{\prime}-M \rho^{\prime}\right)+\frac{\partial}{\partial x} \frac{\partial \nu}{\partial t}+\frac{2}{3} M \frac{\partial^{2} \nu}{\partial x^{2}}\right], \\
\frac{\partial v_{s}}{\partial t}=\frac{1}{m}\left[p^{\prime}-K \xi-R v_{s}\right], \quad \frac{\partial \xi}{\partial t}=v_{s}, \quad \frac{\partial \nu}{\partial t}=\frac{1}{m}\left[(\rho v)^{\prime}-K \eta-R \nu\right], \quad \frac{\partial \eta}{\partial t}=\nu .
\end{gathered}
$$

The boundary filter $\mathcal{F}$ in (27) is defined in (15), with coefficients given in table 3: unless otherwise stated, the $\mathrm{n} 7$ filter is used. The domain is discretized with equidistant grid points spaced by $\Delta x$ and $\Delta y$ in the $x$ - and $y$-directions. Spatial derivatives are calculated using a seven-point fourth-order DRP scheme [11, 15], centered apart from near the boundaries. Time marching is performed using a six-stage fourth-order Runge-Kutta time step [12] of overall time step $\Delta t$. A seven-point sixth order selective spatial filter (filter s7 in table 3) is applied at each time step throughout the domain in both the $x$ - and $y$-directions with strength $S_{\mathrm{F}}$. The point source is numerically implemented as a Gaussian monopole of width $w=\Delta x+\Delta y$, defined by

$$
\boldsymbol{H}=\exp \left\{-\frac{\left(x-x_{s}\right)^{2}+\left(y+y_{s}\right)^{2}}{2 w^{2}}\right\} \frac{\sin (\omega t)}{\sqrt{2 \pi w^{2}}}\left(\begin{array}{c}
1 \\
M \\
0 \\
1
\end{array}\right)
$$

The parameters used for the test cases discussed in the text are given in table 4. 


\begin{tabular}{r|ccc} 
& $\mathrm{A}$ & $\mathrm{B}$ & $\mathrm{C}$ \\
\hline$M$ & 0.5 & 0.5 & 0.4 \\
$\omega$ & 31 & 31 & 10 \\
$y_{s}$ & 0.3 & 0.3 & 0.3 \\
$\delta$ & 0.001 & 0.001 & 0.001 \\
$H$ & 2 & 2 & 2 \\
$L^{-}$ & -4.5 & -4.5 & -4.5 \\
$L^{+}$ & 4.5 & 4.5 & 4.5 \\
$m$ & $\mid$ & 0.01 & 0.08 \\
$K$ & rigid & 10 & 6 \\
$R$ & $\mid$ & 0.75 & 1.6 \\
\hline$\omega_{0}$ & & 31.6 & 8.66 \\
$d$ & & 1.19 & 1.15 \\
$k_{\mathrm{HI}}^{0}$ & & $112.0+105.1 \mathrm{i}$ & $39.5+99.4 \mathrm{i}$ \\
$k_{\mathrm{HI}}^{\delta}$ & & $149.2+87.8 \mathrm{i}$ & $125.0+81.6 I$
\end{tabular}

\begin{tabular}{r|ccc} 
Grid 1 & $\mathrm{A}$ & $\mathrm{B}$ & $\mathrm{C}$ \\
\hline$\Delta x=\Delta y$ & 0.01 & 0.01 & 0.03 \\
$\Delta t$ & 0.006 & 0.006 & 0.017 \\
$\mathrm{CFL}$ & 0.9 & 0.9 & 0.79 \\
PPW $_{\mathrm{a}}$ & 10 & 10 & 12 \\
PPW $_{\mathrm{HI}}$ & & 3.6 & 1.4 \\
$N_{\mathrm{PML}}$ & 15 & 15 & 15 \\
$S_{\mathrm{F}}$ & 0.015 & 0.015 & 0.04
\end{tabular}

\begin{tabular}{r|ccc} 
Grid 2 & $\mathrm{A}$ & $\mathrm{B}$ & $\mathrm{C}$ \\
\hline$\Delta x=\Delta y$ & 0.005 & 0.005 & 0.015 \\
$\Delta t$ & 0.003 & 0.003 & 0.009 \\
$\mathrm{CFL}$ & 0.9 & 0.9 & 0.84 \\
$\mathrm{PPW}_{\mathrm{a}}$ & 20 & 20 & 25 \\
$\mathrm{PPW}_{\mathrm{HI}}$ & & 7.3 & 2.8 \\
$N_{\mathrm{PML}}$ & 15 & 15 & 15 \\
$S_{\mathrm{F}}$ & 0.0075 & 0.0075 & 0.02
\end{tabular}

\begin{tabular}{r|ccc} 
Grid 3 & $\mathrm{A}$ & $\mathrm{B}$ & $\mathrm{C}$ \\
\hline$\Delta x=\Delta y$ & 0.0025 & 0.0025 & 0.003 \\
$\Delta t$ & 0.0015 & 0.0015 & 0.002 \\
$\mathrm{CFL}$ & 0.9 & 0.9 & 0.93 \\
PPW $_{\mathrm{a}}$ & 40 & 40 & 125 \\
PPW $_{\mathrm{HI}}$ & & 14.5 & 14.0 \\
$N_{\mathrm{PML}}$ & 30 & 30 & 25 \\
$S_{\mathrm{F}}$ & 0.00375 & 0.00375 & 0.005
\end{tabular}

\begin{tabular}{r|ccc} 
Grid 4 & $\mathrm{A}$ & $\mathrm{B}$ & $\mathrm{C}$ \\
\hline$\Delta x=\Delta y$ & 0.001 & 0.001 & 0.002 \\
$\Delta t$ & 0.0006 & 0.0006 & 0.0013 \\
$\mathrm{CFL}$ & 0.9 & 0.9 & 0.91 \\
$\mathrm{PPW}_{\mathrm{a}}$ & 101 & 101 & 188 \\
$\mathrm{PPW}_{\mathrm{HI}}$ & & 36.3 & 21.0 \\
$N_{\mathrm{PML}}$ & 75 & 75 & 35 \\
$S_{\mathrm{F}}$ & 0.0015 & 0.0015 & 0.003
\end{tabular}

Table 4: Parameters used for the different test cases. The undamped resonant frequency of the liner is $\omega_{0}=$ $\sqrt{K / m}$, and the damping coefficient is $d=R / \sqrt{4 K m}$ (so that $d>1$ corresponds to over damping). The axial wavenumber of the hydrodynamic (unstable) surface wave for a boundary layer of thickness $\delta$ is denoted $k_{\mathrm{HI}}^{\delta}$. The CFL number is computed using $(1+M) \Delta t / \Delta x$. The minimum number of points per wavelength for an acoustic wave is denoted $\mathrm{PPW}_{\mathrm{a}}$, and for a hydrodynamic surface wave is denoted $\mathrm{PPW}_{\mathrm{HI}}$; in the latter case, since $k_{\mathrm{HI}}$ is complex, the effective wavelength is taken to be $2 \pi /\left|k_{\mathrm{HI}}^{\delta}\right|$.

\section{References}

[1] E. J. Brambley, G. Gabard, Time Domain Simulations using the Modified Myers Boundary Condition, AIAA Paper 2013-2218, doi:10.2514/6.2013-2218, 2013.

[2] T. Melling, The Acoustic Impedance of Perforates at Medium and High Sound Pressure Levels, J. Sound Vib. 29 (1973) 1-65.

[3] A. W. Guess, Calculation of Perforated Plate Liner Parameters from Specified Acoustic Resistance and Reactance, J. Sound Vib. 40 (1975) 119-137.

[4] C. K. W. Tam, L. Auriault, Time-Domain Impedance Boundary Conditions for Computational Aeroacoustics, AIAA J. 34 (5) (1996) 917-923.

[5] S. W. Rienstra, Impedance Models in Time Domain, including the Extended Helmholtz Resonator Model, AIAA Paper 2006-2686, 2006.

[6] E. J. Brambley, Review of Acoustic Liner Models with Flow, in: Proc. Acoustics 2012, Nantes, France, http://hal.archives-ouvertes.fr/hal-00810687, 2012. 
[7] U. Ingard, On the Reflection of a Spherical Sound Wave from an Infinite Plane, J. Acoust. Soc. Am. 23 (3) (1951) 329-335.

[8] M. K. Myers, On the Acoustic Boundary Condition in the Presence of Flow, J. Sound Vib. 71 (1980) 429-434.

[9] W. Eversman, R. J. Beckemeyer, Transmission of Sound in Ducts with Thin Shear Layers Convergence to the Uniform Flow Case, J. Acoust. Soc. Am. 52 (1972) 216-220.

[10] B. J. Tester, Some Aspects of "Sound" Attenuation in Lined Ducts containing Inviscid Mean Flows with Boundary Layers, J. Sound Vib. 28 (1973) 217-245.

[11] C. K. W. Tam, J. C. Webb, Dispersion-Relation-Preserving Finite Difference Schemes for Computational Acoustics, J. Comput. Phys. 107 (1993) 262-281, doi:10.1006/jcph.1993.1142.

[12] C. Bogey, C. Bailly, A Family of Low Dispersive and Low Dissipative Explicit Schemes for Flow and Noise Computations, J. Comput. Phys. 194 (2004) 194-214, doi:10.1016/j.jcp.2003.09.003.

[13] J. Berland, C. Bogey, C. Bailly, Low-dissipation and Low-dispersion Fourth-order Runge-Kutta Algorithm, Computers \& Fluids 35 (2006) 1459-1463.

[14] C. K. W. Tam, J. C. Webb, Z. Dong, A Study of the Short Wave Components in Computational Aeroacoustics, J. Comput. Acoust. 1 (1993) 1-30, doi:10.1142/S0218396X93000020.

[15] J. Berland, C. Bogey, O. Marsden, C. Bailly, High-order, Low Dispersive and Low Dissipative Explicit Schemes for Multiple-scale and Boundary Problems, J. Comput. Phys. 224 (2007) 637-662, doi:10.1016/j.jcp.2006.10.017.

[16] S. W. Rienstra, A Classification of Duct Modes based on Surface Waves, Wave Motion 37 (2003) 119-135, doi:10.1016/S0165-2125(02)00052-5.

[17] N. Chevaugeon, J.-F. Remacle, X. Gallez, Discontinuous Galerkin Implementation of the Extended Helmholtz Resonator Model in the Time Domain, AIAA Paper 2006-2569, 2006.

[18] C. Richter, F. H. Thiele, X. Li, M. Zhuang, Comparison of Time-Domain Impedance Boundary Conditions for Lined Duct Flows, AIAA J. 45 (6) (2007) 1333-1345.

[19] G. Gabard, E. J. Brambley, A Full Discrete Dispersion Analysis of Time-Domain Simulations of Acoustic Liners with Flow, J. Comput. Phys. 273 (2014) 310-326, doi:10.1016/j.jcp.2014.05.004.

[20] E. J. Brambley, Fundamental Problems with the Model of Uniform Flow over Acoustic Linings, J. Sound Vib. 322 (2009) 1026-1037.

[21] C. Richter, J. A. Hay, Ł. Panek, N. Schönwald, S. Busse, F. Thiele, A Review of Time-Domain Impedance Modelling and Applications, J. Sound Vib. 330 (2011) 3859-3873, doi:10.1016/j.jsv.2011.04.013.

[22] E. J. Brambley, M. Darau, S. W. Rienstra, The Critical Layer in Linear-Shear Boundary Layers over Acoustic Linings, J. Fluid Mech. 710 (2012) 545-568, doi:10.1017/jfm.2012.376.

[23] S. Félix, V. Pagneux, Acoustic and Hydrodynamic Modes Generated by a Point Source in a Duct Carrying a Parallel Shear Flow, in: Proc. 19th International Congress on Acoustics, Madrid, 2-7 September, 2007.

[24] D. Marx, Numerical Computation of a Lined Duct Instability using the Linearized Euler Equations, AIAA J. 53 (8) (2015) 2379-2388, doi:10.2514/1.J053746.

[25] E. J. Brambley, A well-posed boundary condition for acoustic liners in straight ducts with flow, AIAA J. 49 (6) (2011) 1272-1282, doi:10.2514/1.J050723.

[26] S. W. Rienstra, M. Darau, Boundary-Layer Thickness Effects of the Hydrodynamic Instability along an Impedance Wall, J. Fluid Mech. 671 (2011) 559-573.

[27] G. Gabard, A Comparison of Impedance Boundary Conditions for Flow Acoustics, J. Sound Vib. 332 (2013) 714-724, doi:10.1016/j.jsv.2012.10.014. 
[28] N. Peake, On the Behaviour of a Fluid-Loaded Cylindrical Shell with Mean Flow, J. Fluid Mech. 338 (1997) 387-410.

[29] E. J. Brambley, N. Peake, Stability and Acoustic Scattering in a Cylindrical Thin Shell Containing Compressible Mean Flow, J. Fluid Mech. 602 (2008) 403-426.

[30] Y. Aurégan, M. Leroux, Experimental Evidence of an Instability over an Impedance Wall in a Duct with Flow, J. Sound Vib. 317 (2008) 432-439.

[31] D. Marx, Y. Aurégan, H. Bailliet, J.-C. Valière, PIV and LDV Evidence of Hydrodynamic Instability over a Liner in a Duct with Flow, J. Sound Vib. 329 (2010) 3798-3812, doi:10.1016/j.jsv.2010.03.025.

[32] S. H. Arzoumanian, Stability of Fluid-Loaded Structures, Ph.D. thesis, University of Cambridge, URL http://www.dspace.cam.ac.uk/handle/1810/243913, 2011.

[33] E. J. Brambley, Surface Modes in Sheared Boundary Layers over Impedance Linings, J. Sound Vib. 332 (2013) 3750-3767, doi:10.1016/j.jsv.2013.02.028.

[34] Y. Aurégan, R. Starobinski, V. Pagneux, Influence of Grazing Flow and Dissipation Effects on the Acoustic Boundary Conditions at a Lined Wall, J. Acoust. Soc. Am. 109 (2001) 59-64.

[35] E. J. Brambley, Acoustic Implications of a Thin Viscous Boundary Layer over a Compliant Surface or Permeable Liner, J. Fluid Mech. 678 (2011) 348-378, doi:10.1017/jfm.2011.116.

[36] D. Khamis, E. J. Brambley, The Effective Impedance of a Finite-Thickness Viscothermal Boundary Layer Over an Acoustic Lining, AIAA Paper 2015-2229, doi:10.2514/6.2015-2229, 2015.

[37] Y. Renou, Y. Aurégan, Failure of the Ingard-Myers Boundary Condition for a Lined Duct: An Experimental Investigation, J. Acoust. Soc. Am. 130 (2011) 52-60, doi:10.1121/1.3586789.

[38] D. Marx, Y. Aurégan, Effect of Turbulent Eddy Viscosity on the Unstable Surface Mode above an Acoustic Liner, J. Sound Vib. 332 (2013) 3803-3820, doi:10.1016/j.jsv.2013.02.005.

[39] E. J. Brambley, G. Gabard, Reflection of an Acoustic Line Source by an Impedance Surface with Uniform Flow, J. Sound Vib. 333 (2014) 5548-5565, doi:10.1016/j.jsv.2014.05.026.

[40] F. Q. Hu, A Stable Perfectly Matched Layer for Linearized Euler Equations in Unsplit Physical Variables, J. Comput. Phys. 173 (2001) 455-480, doi:10.1006/jcph.2001.6887.

[41] D. C. Pridmore-Brown, Sound Propagation in a Fluid Flowing Through an Attenuating Duct, J. Fluid Mech. 4 (1958) 393-406.

[42] D. Khamis, E. J. Brambley, Viscous effects on the acoustics and stability of a shear layer over a non-rigid wall, submitted to J. Fluid Mech., 2016.

[43] Y. Renou, Y. Aurégan, On a Modified Myers Boundary Condition to Match Lined Wall Impedance Deduced from Several Experimental Methods in Presence of a Grazing Flow, AIAA Paper 2010$3945,2010$. 